\title{
Preattentive texture segmentation: The role of line terminations, size, and filter wavelength
}

\author{
BARTON S. RUBENSTEIN and DOV SAGI \\ The Weizmann Institute of Science, Rehovot, Israel
}

\begin{abstract}
The $\triangle / K$ texel pair represents a dilemma in texture discrimination because, despite having the same component orientations, discrimination is still possible (Julesz, 1981), showing a performance asymmetry. Other possible element properties that could influence this task are line terminations, closure, and the size of these elements. We found that line terminators are critical for the task; however, results from double-task experiments indicated that terminator-based discrimination requires the use of attention. When attention is not available for the task, "size" of the elements (with the $K$ considered slightly larger) seems to be critical for this discrimination and for the asymmetric performance. To generalize the concept of "size" to textures in general, further experiments were performed with textures of different-sized elements. Results showed, as past literature has indicated, that there is a performance asymmetry, with the larger of the elements being more visible when in the foreground. This asymmetry was additionally shown to reverse itself (i.e., the smaller element became the more visible) as the scale of the elements increased (while interelement distance remained fixed). A filter analysis was developed in order to measure the apparent size of these elements within textures (texsize), defined as the response weighted average of the filter wavelength, $\bar{\lambda}$, for a group of elements. The calculation of $\bar{\lambda}$ was attained by introducing a nonlinearity after the second stage of filtering (or spatial averaging of filter responses). This analysis showed high correlation between the texture with the larger $\bar{\lambda}$ and the more visible texture. On the basis of this correlation, a wavelength-dependent noise is proposed, having more internal noise for low-spatial-frequency filters and less for high-spatial-frequency filters.
\end{abstract}

An early attempt to explain texture discrimination was based on determining joint probability distributions of two luminance levels (Julesz, Gilbert, Frisch, \& Schepp, 1973). It was shown that, although regions with iso-first-order statistics (luminance) could be easily discriminated, those with expected iso-second-order statistics (energy) could not. Later, many counterexamples were discovered (such as the $\Delta / K$ pair), and consequently, Julesz $(1981,1984,1986)$ developed the texton theory to define stimulus features that could give rise to spontaneous discrimination. These features (textons) included blob-like objects having particular orientations, lengths, widths, color, and intensity, with discrimination possible for adjacent texture regions differing in the density of these textons. This approach to understanding global luminance structure seemed well correlated with psychophysical results of texture discrimination tasks; however, it provided little intuition as to how such primitives could be extracted from arbitrary or natural images.

An alternative theory purported that properties defining a region's homogeneity could be estimated using spatially localized operators, like those of the early visual system (Beck, Sutter, \& Ivry, 1987; Bergen \& Adelson, 1988; Caelli,

This work was supported by the Basic Research Foundation administered by the Israel Academy of Sciences and Humanities. The authors would like to thank Yoram Bonneh for his comments and Shereen Rubenstein for her assistance with the editing of the manuscript. Correspondence should be addressed to D. Sagi, Department of Neurobiology, Brain Research, The Weizmann Institute of Science, Rehovot 76100, Israel (e-mail: dubi@nisan.weizmann.ac.il).
1985). These operators were defined to be contrast sensitive and selective to a particular bandwidth of orientations and spatial frequencies. A Gabor filter is an example of such an operator, and it has been shown to be quite similar to simple cells in the cat striate cortex (see Daugman, 1985). On the basis of computing local energy, these operators are able to differentiate between various textural regions (Caelli, 1985; Landy \& Bergen, 1991) and can predict human performance (Fogel \& Sagi, 1989; Malik \& Perona, 1990; Rubenstein \& Sagi, 1990). A second stage of filtering, combining spatially nearby filter responses, has also been psychophysically supported (Cannon \& Fullenkamp, 1991; Chubb, Sperling, \& Solomon, 1989; Rubenstein \& Sagi, 1993; Sagi \& Hochstein, 1985). More recently, relative phase information (both one and two dimensional) between neighboring textures has been shown to be a critical property for discrimination (Hoffman \& Hallett, 1993). Filters can also model this behavior, because even and odd filters can encode both phase and energy properties.

Several researchers (Beck, 1973; Gurnsey \& Browse, 1987; Julesz, 1981; Treisman, 1985) observed a performance asymmetry when certain target and background textures were exchanged. Although the same textural border exists in both stimuli, using one texture element in the background makes discrimination of the target easier than in the reverse case. The implication of this behavior is that texture discrimination is not merely the result of local computations (one that localizes textural gradients); otherwise, human performance would be the same regardless of which texture is in the background. Rather, there must be some 
global calculations that contribute to the task. Rubenstein and Sagi (1990) were able to predict human performance asymmetries by creating a multistage model, using first stage linear filters, based on signal detection theory. They claimed that a foreground's visibility was limited by spurious energy gradients in the background that act to confuse the observer. This background "noise" was hence defined by spatial variability of the texture in the background. Studying many elements, they showed that an element's energy varies across the orientation spectrum and that elements may have different profiles and different amounts of energy variability. Thus, for stimuli with randomly oriented elements, adjacent like elements could produce energy gradients of different magnitudes, depending on this variability. Using psychophysical results of Gurnsey and Browse (1987, using stimuli with randomly oriented elements), they predicted that the background element with more energy variability across orientation would give rise to reduced visibility of the foreground. Then, according to signal detection theory, performance was predicted by computing the probability that the signal (foreground-tobackground energy gradients) was larger than the spurious gradients in the background. The correlation of model predictions to psychophysics was quite strong, with only one interesting exception. Different from other pairs of elements used, the $\Delta$ and $K$ produced energy profiles across orientation in contradiction to the above argument. Specifically, the $\Delta$ was shown to have more energy variability than the $K$, as seen by the curves of Figure 1 at low spatial frequencies. Thus, the Rubenstein and Sagi model would predict a more visible $\Delta$ in the foreground, while $\Delta \mathrm{s}$ in the background would produce spurious gradients and thus limit the visibility of $K$. Psychophysics, however, showed the opposite asymmetry, with a more visible $K$. Gurnsey and Browse actually used a $\Delta$ and $K$ that were slightly flattened, producing much less difference in energy variability across orientation; however, although performance was low (as the theory would predict), there was a slight asymmetry reported. This exception leaves open the possibility that other stimulus features exist, which can at times dominate over the energy variability described above. It should be noted that the term feature is used only to describe stimulus characteristics, while filter-based models (e.g., Rubenstein \& Sagi, 1990) generally act independently of this designation (an exception is when the feature described relates to a line's orientation).

The first important property of the $\Delta$ and $K$ is that they produce texture ensembles with iso-second-order statistics (Caelli \& Julesz, 1978). Thus, by averaging filter response across orientation (or space, in the case of textures with randomly oriented texture elements), identical energy values are produced. Other distinguishing properties of these elements (in comparison with the other pairs used by Gurnsey and Browse) are differences in the number of line terminations (zero and three) and that one of the elements is a closed object. Also, the size (when defined as the minimal enclosing ellipse) of the $K$ may perhaps be larger than that of the $\triangle$. According to Gurnsey and Browse (1989), elements of different sizes can be discriminated with the
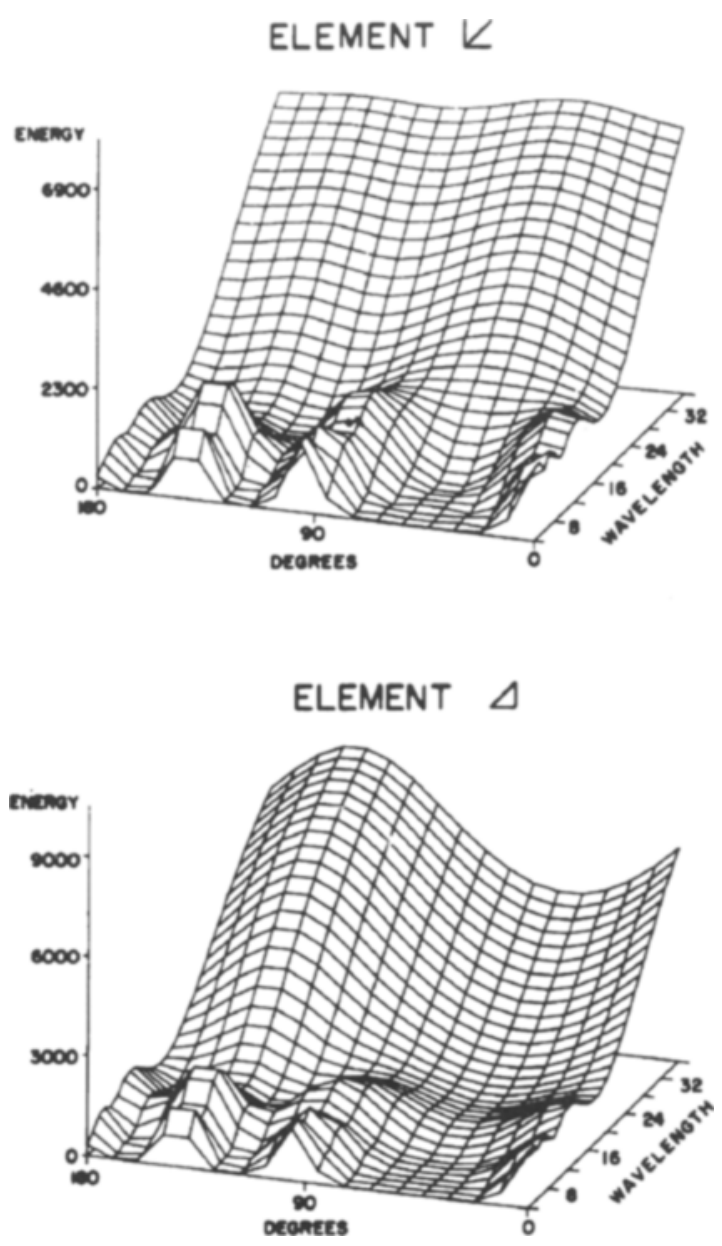

Figure 1. Space invariant energy responses for the $\Delta$ and $K$ (see Equations A1-A6 in the Appendix). Each vertex of the threedimensional curve represents a response of a filter with a particular orientation and spatial frequency. Notice that, for low spatial frequencies (back of curves), the $\Delta$ has more variability across the orientation spectrum than that of the $K$.

larger of the elements being more visible when in the foreground. All of these stimulus properties have been purported to be extracted by and somehow encoded in the visual brain; however, there has been some controversy as to which level in the visual process extraction occurs. In understanding the lower levels of visual processing, Neisser (1967) separated visual processing into two modules, with a preattentive process, one that acts on the whole image at once (in parallel), followed by an attentive process that can process only one selected portion of the image at a time (serial). The attentive process has been shown to occur with or without eye movements, with shifts of focus onto different portions of an image guided by information provided by the already completed preattentive process. The attentive process, sometimes described as a top-down process, is one that is concerned with the identification of objects, whereas the preattentive system appears to be more of a bottom-up process and can only detect inhomogeneities in an image. 
The objective of this work was to determine the importance of two stimulus properties: the size of the microelements in a texture and line terminations. Toward this goal, elements with different sizes and different interelement distances were used. As will be seen, the interelement distance can be critical to the task, since varying the ratio of the size of an element to the interelement distance can at times reverse the performance asymmetry (i.e., the smaller element becomes the more visible). A filter analysis was also developed in order to estimate a texture element's apparent size, or texsize, defined as the response weighted average of the filter wavelength, $\bar{\lambda}$,

$$
\bar{\lambda}=\frac{\sum_{\lambda} \lambda \cdot R(\lambda)}{\sum_{\lambda} R(\lambda)},
$$

with $R(\lambda)$ being the space averaged response of a filter with wavelength $\lambda$ (see the Appendix for details). Note that because this measure includes more than one individual element, texsize not only represents the element's size but also the spatial-frequency content of the texture region, including the effect of interelement distance. This texsize measure appears to be more representative of the visual system, in that an element's properties are not only a function of its own inherent properties but also those of the immediate neighborhood that surrounds it. Consequently, stimuli were designed with texsize in mind, with psychophysical results indicating that the texture (numerous neighboring elements of the same type) with the larger $\bar{\lambda}$ was always the more visible. A filter wavelength-dependent noise is proposed that can account for this performance asymmetry. Using the framework of signal detection theory implemented in the Rubenstein and Sagi (1990) model, along with an additional nonlinearity implemented after the second filtering stage, low-spatial-frequency filters are speculated to have more internal noise than highspatial-frequency filters. This idea gives rise to a large $\bar{\lambda}$ background texture producing more spurious gradients and thus reducing visibility of the smaller $\bar{\lambda}$ texture in the foreground. On the basis of this analysis, additional experiments were performed to determine at which processing level texsize $(\bar{\lambda})$ and line terminations are extracted, with results showing the former to be preattentive, while line terminations use attentive resources.

\section{METHOD}

\section{Observers}

Three observers participated in the experiments, one of which was one of the authors (B.R.). The others were high school students paid to participate and were naive as to the purpose of the experiments. All had normal vision.

\section{Apparatus}

The stimuli were presented in a dark environment on a HewlettPackard 1310B oscilloscope (P31 phosphor). The oscilloscope was driven by custom-designed hardware allowing for real-time control of the stimulus properties. Screen resolution was $1,024 \times 1,024$ pixels, and a viewing distance of $150 \mathrm{~cm}$ resulted in a display subtending approximately an $11^{\circ} \times 11^{\circ}$ visual angle $\left(1.07^{\circ}\right.$ for each $100 \mathrm{pix}-$ els). The experiments and graphic device were controlled by a Sun3/160 Workstation.

\section{Stimulus Design and Procedure}

A four-alternative forced-choice (4AFC) paradigm was used, with stimuli composed of a background, a matrix of elements, and a smaller matrix of foreground elements placed in one of the four quadrants. Each element was randomly jittered up to 6 pixels $\left(3.8^{\prime}\right)$ in any direction from the vertex of the matrix that it was originally assigned. This was done to remove global patterns that might arise from elements perfectly aligned. Many of the experiments used randomly oriented elements, and, in such cases, 10 different orientations were used (steps of $36^{\circ}$ ). For elements of fixed orientation, elements had an angle base of $22^{\circ}$, so as to offset any global patterns that might arise from the vertical and horizontal arrangement of the entire matrix. Elements were presented at different scales, and, accordingly, we arbitrarily assigned elements whose line segments fit tightly within a $20 \times 20$ square pixel space as $100 \%$ scale. In this case, line elements (with luminance of $35 \mathrm{~cd} / \mathrm{m}^{2}$ in an otherwise dark background) were 1 pixel wide $\left(0.64^{\prime}\right)$, and bright square blobs that were added to the elements were $5 \times 5$ pixels $\left(3.2^{\prime}\right.$ on a side). Consequently, if the chosen scale was larger, everything increased in size, including the square blob size, line width, and length.

Three different types of stimuli were used. The first type (used in Experiments 1, 4, 8, and 10) used a $15 \times 15$ matrix of elements, with a foreground, a $3 \times 3$ matrix, placed within one of four quadrants with a slightly randomized position (see Figure $2 a$ ). Interelement distance was on average 64 pixels. The average eccentricity of the center of the foreground region was $4.1^{\circ}$, thus producing a peripheral foreground, small-spacing stimulus. The second stimulus type, peripheral foreground, large-spacing stimulus (Experiments 1 and 6), used an interelement distance of 128 pixels. The stimulus was a $7 \times 7$ matrix of elements with a $2 \times 2$ foreground region also randomly positioned within the respective quadrant (see Figure 2b). On average, the eccentricity of this foreground was the same as the previous stimulus type. Random positioning of the foreground region was implemented to increase the level of difficulty of the task and, thus, to collect data that were neither too difficult nor too easy. The third stimulus type (Experiments 2, 3, 5, 7, and 9) was composed of a $8 \times 8$ matrix of elements and a $3 \times 3$ foreground, whose inside corner was always next to the center of the stimulus (see Figure 4a). Interelement distance was 100 pixels, producing a near-foveal foreground, medium-spacing stimulus.

Temporal sequence. A backward masking paradigm was used. Presenting a mask at some period after the presentation of the stimulus acts to erase (or overwrite) the stimulus in the retina and thus reduce its accessibility. The mask for all experiments was similar to the stimulus except the elements were offset in both $x$ and $y$ directions by half of the interelement distance (see Figure 4d). The type of element was selected at random from the two stimulus elements with a $50 \%$ probability (with the exception of Experiment 1, which used one element type composed of the superposition of the two stimulus elements). This was implemented so as to avoid summation of superimposed stimulus and mask elements, which, for small interstimulus intervals (ISIs), can give rise to unwanted masking effects. The mask of Experiment 1 was composed of elements that were a hybrid of the two stimulus elements (see Figure $2 \mathrm{f}$ ). Both mask types have been used extensively in the literature and appear to produce similar effects on stimulus visibility. During the experiments, a fixation cross was presented in the center of the screen so as to center the observer's attention. Upon pressing a key, the trial began with a blank display $(100 \mathrm{msec})$, followed by the stimulus $(20 \mathrm{msec})$, an ISI (a blank screen whose range was between 0 and $500 \mathrm{msec}$ ), the mask ( $100 \mathrm{msec})$, and, finally, a blank screen during which time the observer had to answer on the keypad the quadrant in which the foreground was presented (i.e., $0,1,2$, or 3 ). If the observer did not see any foreground (even though it always was present), he/she was asked to randomly choose an answer. Auditory feedback, by means 
(a)
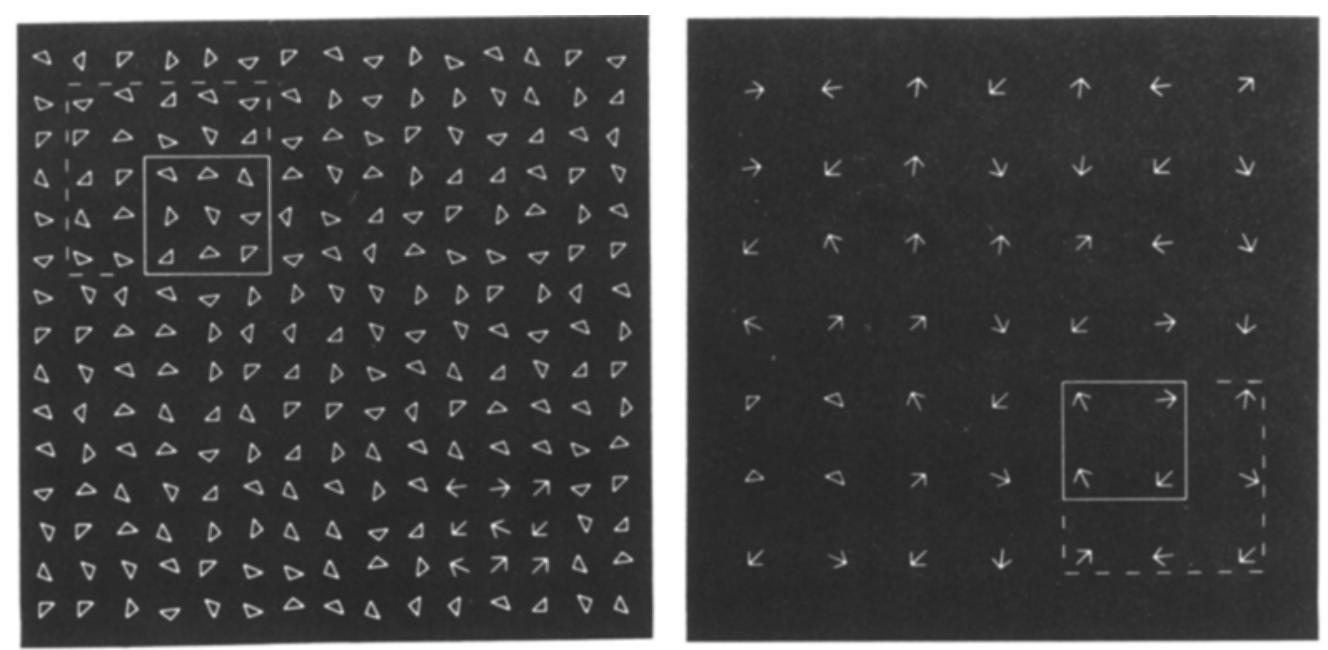

(b)
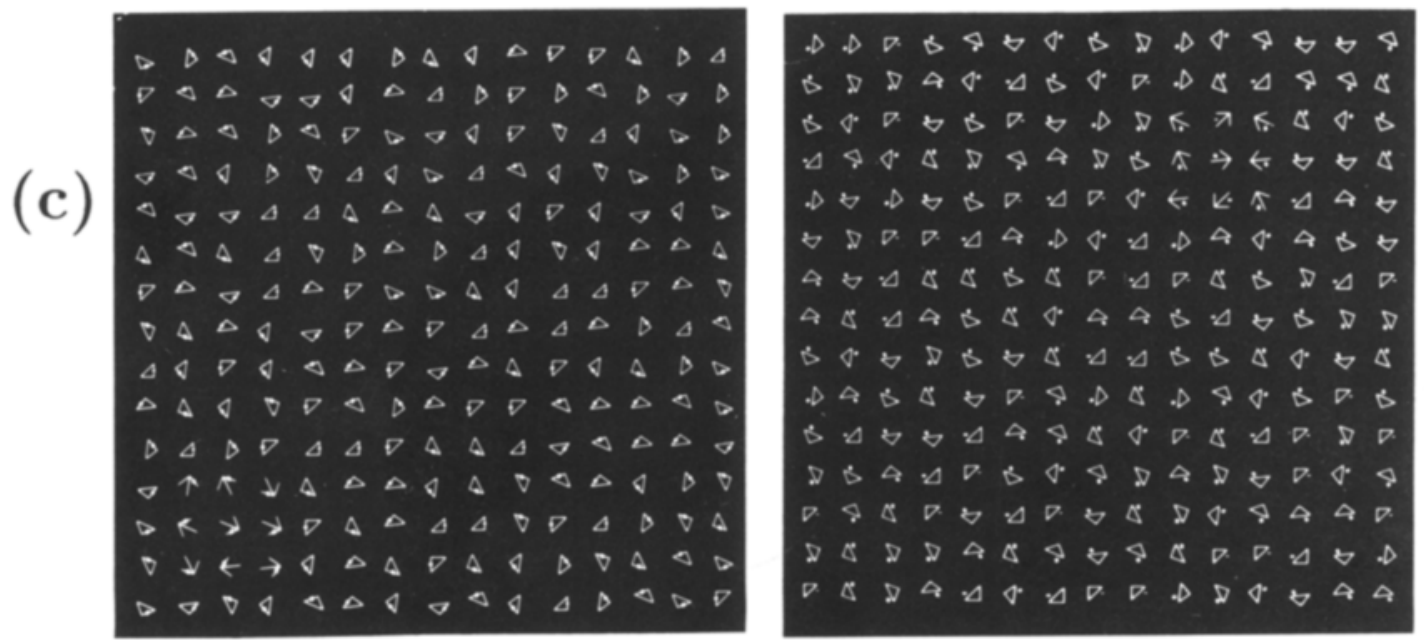

(d)
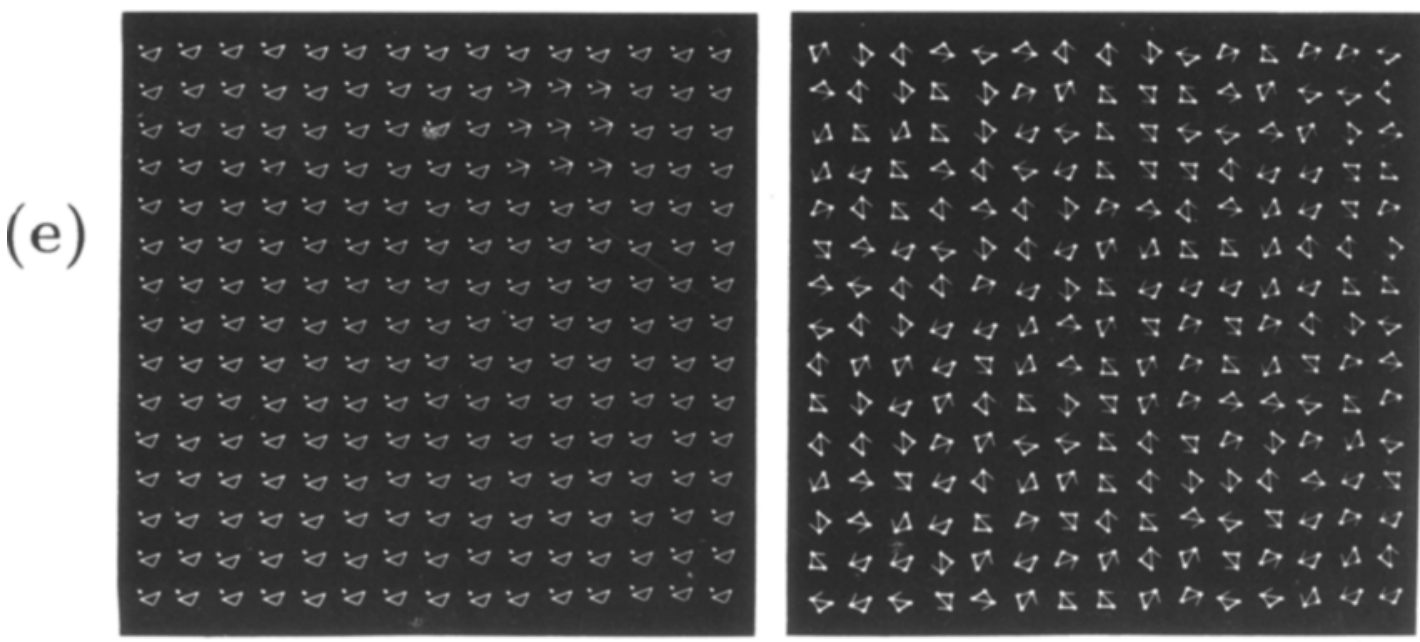

Figure 2. Sample stimuli used in Experiment 1. Three types of stimuli were used-peripheral foreground, smallspacing, and randomly oriented elements (panels a, c, and d), peripheral foreground, large-spacing, and randomly oriented elements (panel b), peripheral foreground, small-spacing, and elements of fixed orientation (panel e)-and a mask (panel $f$ ) composed of elements that are a hybrid of the stimulus elements. For clarity, in panels a and $b$, there is also a box shown, defining the size of the foreground. The dotted box represents the area in which the foreground can be randomly placed. (Note here that the actual foreground elements are not in the boxes.) 
of a keyboard bell, was provided upon the observer's error immediately after the response.

Staircase method. Difficulty of the task was measured using a staircase method described by Levitt (1971). In particular, the ISI was altered during the course of a block of trials according to the following criteria: for every incorrect response, the ISI was increased by $2 \mathrm{~dB}(25.89 \%)$, and, for every three consecutive correct answers, the ISI was reduced by the same percentage. This change of ISI is denoted as a step. On the basis of criteria for statistical stability defined by Levitt, each block continued until there were at least six to eight reversals in the staircase (i.e., an increase of the ISI followed by a decrease, or vice versa), which could last anywhere from 32 to 50 trials. Then, by averaging the ISI values at the reversals, a $79.4 \%$ threshold value was produced. With the graphic system used, frame time was as low as $1 \mathrm{msec}$, providing good resolution for this type of staircase. Also, for some difficult tasks, ISIs exceeding $500 \mathrm{msec}$ were required, indicating an attentive serial task. In these cases, the block was aborted if the observer could not succeed to return below the 500 -msec mark or if the ISI exceeded $1,000 \mathrm{msec}$. For these tasks, preblock ISI settings were $300 \mathrm{msec}$.

During a block, only one type of stimulus (same elements in their respective foreground and background) was used, with each trial varying by the quadrant chosen for the foreground, the foreground position within that quadrant, the positional jitter of each of the elements, and the ISI. Before each block, a sample stimulus was presented in order for the observers to know in advance what type of stimulus would be shown. This was done to reduce stimulus uncertainty. For each stimulus, at least three and up to seven blocks were performed, all similar with the exception of the initial ISI setting. ISI was set before each block to equal (actually rounded up to the nearest $10 \mathrm{msec}$, but this difference is insignificant) the threshold ISI of the previous block for the same stimulus. This original ISI setting only shortens the length of the block; with a large initial ISI of $180 \mathrm{msec}$, it would take three steps (nine trials) to arrive at $76 \mathrm{msec}$, whereas starting with $70 \mathrm{msec}$ would remove these unnecessary steps (assuming the eventual threshold is around $70 \mathrm{msec}$ ). Experiments were performed in chronological order as presented here. For each experiment, blocks were randomly mixed. Each observer was limited to $1 \mathrm{~h}$ (a session) of experimentation per day so as to offset the effects of fatigue. Also, the observers were allowed to practice for a few sessions before the experiments began in order to acclimate themselves to the task and, more importantly, to learn the task to a point that they could reproduce similar results (behavior).

Double-task method. In addition to this paradigm, a double task was implemented in Experiment 3. Double-task experiments can be useful when trying to determine whether a task is preattentive or attentive. Braun and Sagi (1990) showed that a preattentive (parallel) task will not suffer in performance with the introduction of a second attentive task, whereas an attentive (serial) task will suffer. These results support a preattentive system that acts independently of the attentive system and a load-sharing attentive system that must divide its processing power, but at the expense of reduced efficiency. Accordingly, we used the same difficult attentive task, using a V-like element (with one arm shorter than the other, 24 and 34 pixels in length) placed in the center of the original stimulus (see Figure 4a). The task was to determine whether the longer arm was in a clockwise or counterclockwise direction from the shorter arm, with the element randomly oriented from trial to trial. Following the stimulus, an additional mask for this central task was introduced, with a constant ISI of $150 \mathrm{msec}$ (see Figure 4d). Depending on the ISI of the regular mask, both masks were either coincident or presented at different moments. After the presentation of the stimulus and masks, the observer first had to respond to the central task ( 1 for clockwise, 2 for counterclockwise orientation), followed by responding to the 4AFC task. The observers were instructed to perform the central task with a high degree of accuracy to ensure that they were concentrating on the task. Blocks that produced performance levels less than $85 \%-100 \%$ were not used. Because of the extreme difficulty of this central task, the observers took up to 2 days before reaching the above-mentioned criteria of the double-task experiment.

\section{THE $\triangle I K$ PUZZLE: WHICH FEATURES ARE CRITICAL TO THE TASK?}

\section{Experiment 1: Texsize Is Important for $\triangle I K$ Discrimination}

The central question in designing Experiment 1 was to determine what features are inherent to the $\Delta$ and $K$ that allow them to be discriminable. Since both have the same spatial frequency (averaged across orientations) power spectra, discrimination cannot be based on global (average) energy differences if the elements are randomly oriented in the display. As mentioned earlier, Rubenstein and Sagi (1990) showed that energy variability across the orientation spectrum will give rise to spatial variability (gradients) and, as Figure 1 shows, the $\Delta$ has more variability and thus would be predicted as the pop-out (i.e., more visible when in the foreground); however, the reverse is true. Hence, there must be some other more dominant features that allow the $K$ to be the more visible foreground. At first glance, stimulus properties, such as line terminations (see Julesz, 1984, 1986) or closure (Kovács \& Julesz, 1993; Treisman, 1985), might be important. However, until now, neither have been shown to be preattentively extracted. The size of an element was also considered to be a critical property for discrimination.

The elements chosen in this experiment were designed to determine whether the size of an element was crucial to the task. The bottom of Figure 3 shows eight pairs of elements, each of which represents a pair of elements used in a stimulus. The first six pairs were composed of $\Delta \mathrm{s}$ and $\mathrm{Ks}$, whose line segments fit perfectly within a square. Square blobs were also added to elements in each pair (except Pair 1.1) at identical locations. According to methods described by Caelli, Julesz, and Gilbert (1978), blobs were placed in such a way that elements in a pair continued to have the same second-order statistics. This was done to ensure that the task would remain energy independent. Thus, pair elements remained isoenergy, while the texsize varied. It should be noted that pair elements are isoenergy only when they are randomly oriented and not of fixed orientation. Pairs 1.7 and 1.8 were also used, and their importance is tied to the following experiments. Three different stimulus conditions were used, all with peripheral foregrounds. For randomly oriented elements, both small and large interelement distances were used; for elements of fixed orientation, small spacing was implemented. Elements were scaled to fit within a $25 \times 25$ pixel space.

Data are depicted in Figure 3 for 3 observers. As for all the data graphs of this paper, ISI threshold (in milliseconds) is represented on the vertical axis, and all element pairs are placed along the horizontal axis. Performance for stimuli with a particular element in the foreground is represented by the rectangle above that element (with the partner element representing the background element). Each rectangle represents performance averaged across all blocks performed on that particular stimulus, and the T-bars rep- 
Experiment 1

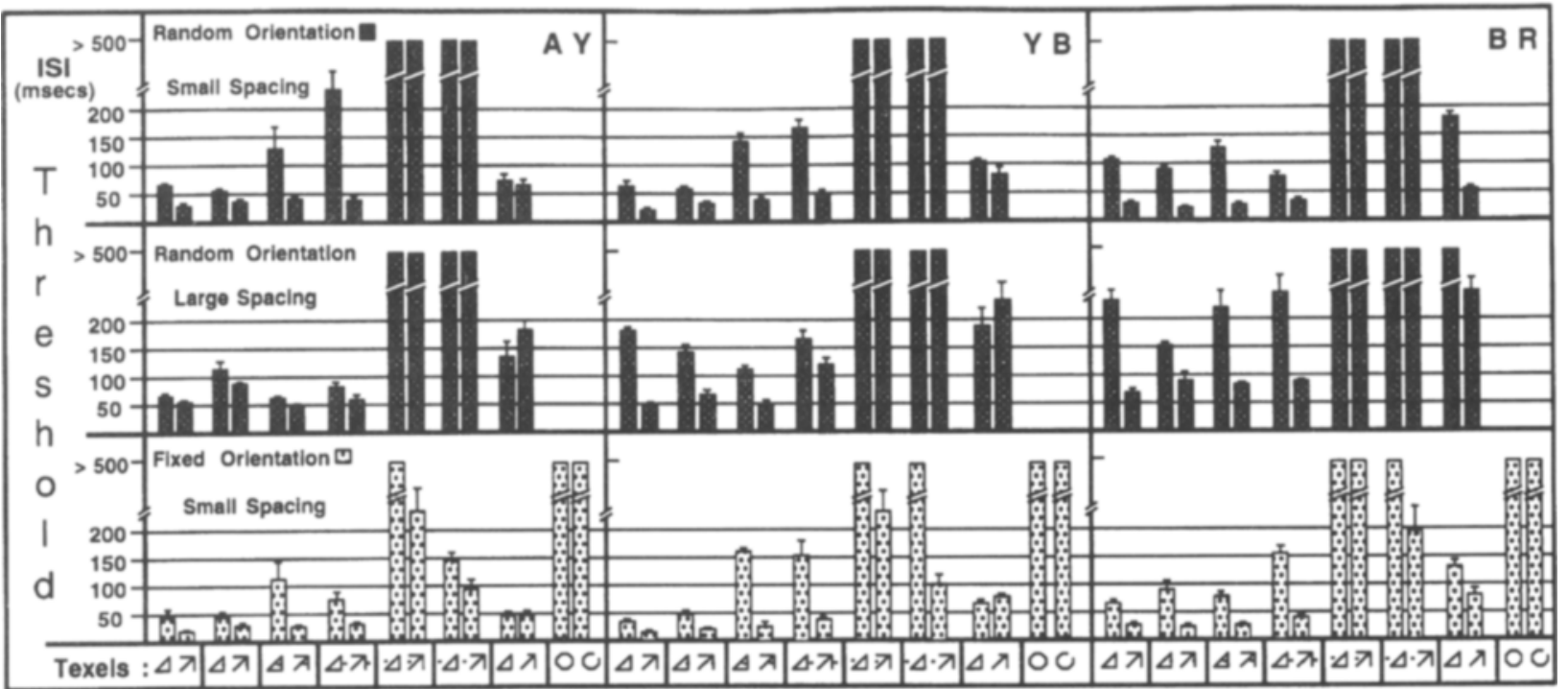

\begin{tabular}{|c|c|c|c|c|c|c|c|c|}
\hline $\begin{array}{l}\text { Texel: } \\
\text { Pair: }\end{array}$ & $\triangle_{1.1} 7$ & $\Delta 7$ & $\Delta_{1.3} 7$ & $\triangle \cdot 7 \cdot$ & 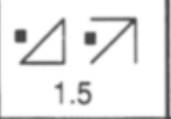 & $\cdot \triangle_{1.6} \cdot 7$ & $\left.\triangle_{1.7}\right\rceil$ & 1.8 \\
\hline$\lambda:$ & $58.1 \quad 59.3$ & $57.5 \quad 58.6$ & $57.1 \quad 58.1$ & $57.8 \quad 58.5$ & $59.1 \quad 59.0$ & $59.2 \quad 59.1$ & $58.1 \quad 58.3$ & $60.1 \quad 55.3$ \\
\hline
\end{tabular}

Figure 3. Psychophysical data of Experiment 1 are depicted at top for 3 observers and eight different pairs of elements. Three types of stimuli were presented: randomly oriented elements and small interelement distance (top third), randomly oriented elements and large interelement distance (middle third), and elements of fixed orientation and small interelement distance (bottom third). Above each element is a rectangle showing the performance threshold (measured with the interstimulus interval, ISI) when that element was in the foreground and the other element in the pair was in the background. Each rectangle represents performance averaged across blocks for each observer, with T-bars indicating the corresponding standard errors. Notice that, for Pairs 1.1-1.4, placement of the square blobs contributed little to the task, whereas, for Pairs 1.5 and 1.6, the task suddenly became quite difficult. At bottom, depicted with each element is the response weighted average of the filter wavelength, $\bar{\lambda}$ (expressed in pixels). Half- $\bar{\lambda}$ values represent the excitatory annuli of such filters and should be approximately the same as the widths of the elements (i.e., without blobs, 25 pixels). Details of filter analysis are presented in the Appendix.

resent the standard error. For Pairs 1.1-1.4, all data indicate that the $K$ was the more visible of the pair, in agreement with Gurnsey and Browse (1987). However, for Pairs 1.5 and 1.6 , thresholds increased noticeably above those of the rest of the pairs, particularly for random-orientation conditions. Upon inspection of Pairs 1.4 and 1.5, with blobs slightly offset from the elements, it appears that these elements are quite similar. However, data indicate that some critical feature of the elements in Pair 1.5 has been masked. If one were to consider the size of an element an important property, it might appear that introducing a blob outside of the $\Delta$ and next to its vertical line segment (Pair 1.4) would not change its apparent size (defined as the minimal enclosing ellipse), whereas placing the blob in an otherwise empty space (Pair 1.5) might. To determine the apparent size of these elements more quantitatively, a filter analysis was developed (see the Appendix). Calculations for the isoenergy pairs (Pairs 1.1-1.6) indicated that the response weighted average of the filter wavelength, $\bar{\lambda}$, or texsize, corresponded very closely to the above, more intuitive, apparent size (see bottom of Figure 3). Specifically, for the very difficult tasks (i.e., Pairs 1.5 and 1.6) the correspond- ing $\bar{\lambda}$ values are practically identical (for each pair, values are separated by 0.1 pixel units). For the easier tasks using Pairs 1.1-1.4, differences between $\bar{\lambda}$ s for each pair are at least seven times larger. Thus, discriminability can be correlated to similarity of $\bar{\lambda}$ values. It should be noted that, although the actual differences between $\bar{\lambda}$ values for Pairs $1.1-1.4$ are only $1 \%-2 \%$, texsizes can still be differentiated. This small difference could either imply a high sensitivity of the visual system or simply indicate a texsize measure that does not incorporate other variables that could enlarge this difference.

Comparing random-orientation stimuli of small and large interelement distances, one finds similar data. This indicates that, for elements of this scale, relative to the interelement distance, there is little interaction between adjacent elements (assuming that interactions are range dependent). For stimuli with elements of fixed orientation, there were also similar data, except for Pairs 1.5 and 1.6. In these cases, thresholds became less than the 500-msec ceiling, with the $K$ continuing to be the more visible of the pair. These data could be caused by the blobs being positioned in a more orderly matrix-like arrangement, which could 

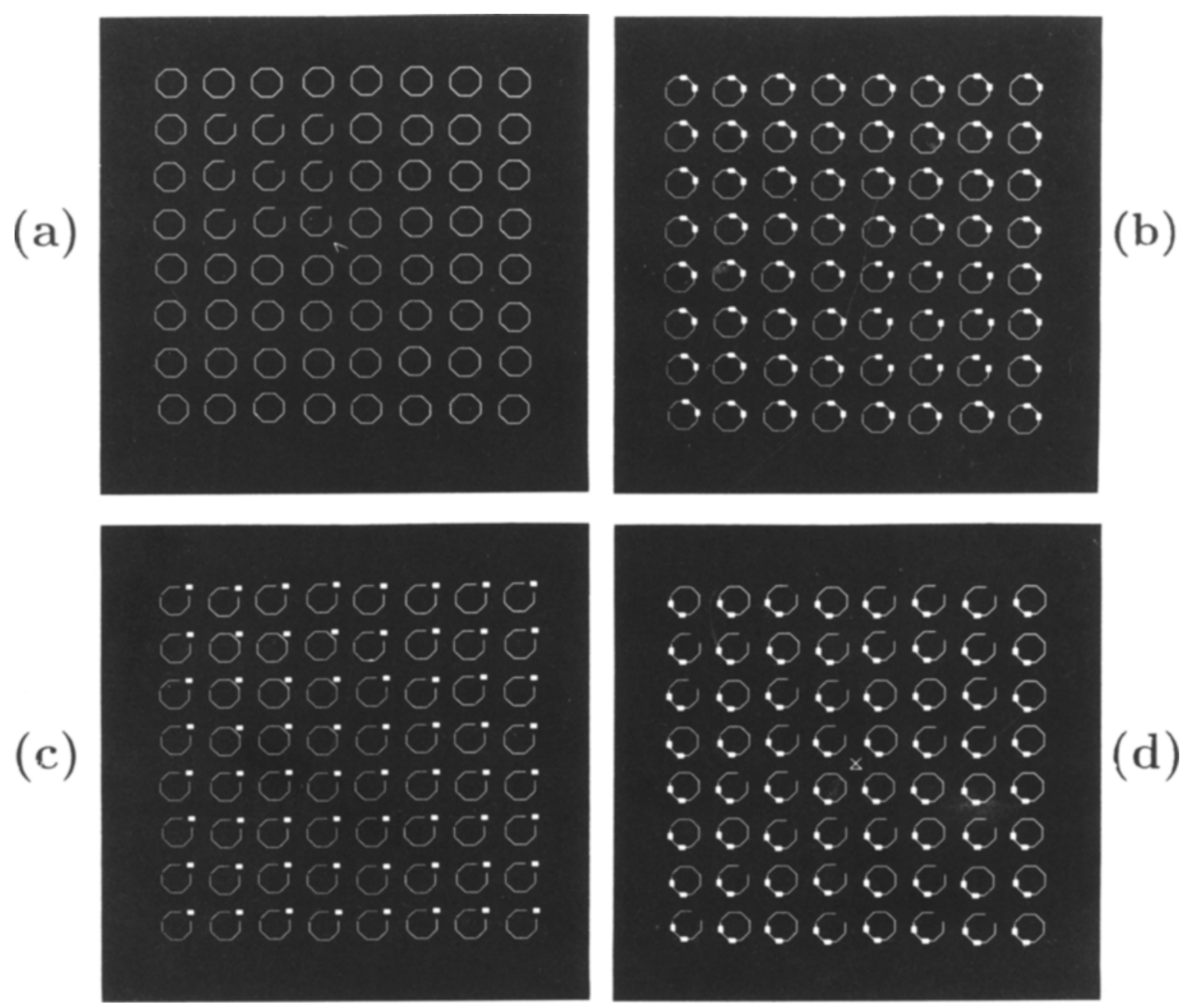

Figure 4. Sample stimuli used in Experiment 2. A near-foveal foreground, medium-spacing stimulus was used. Stimuli shown here are for Pair 2.1 (panel a), Pair 2.2 (panel b), Pair 2.4 (panel c), and a typical mask for Pair 2.3 (panel d), using a $50 \%$ combination of the elements. For double-task experiments (used in Experiment 3), a central element is added to the stimulus (panel a), along with its corresponding mask element (panel d).

give rise to some sort of transparency effect (i.e., stable noise in the background that can ultimately be ignored). This effect is perhaps even stronger for Pair 1.6, with the blobs even farther away from the original elements, and is thus reflected in the data by lower thresholds (relative to Pair 1.5). In contrast, for randomly oriented elements, this "blob matrix" is not apparent, and blobs tend to attach themselves to their respective elements. Pair 1.7 was used to determine if reducing the size of the $K$, by shortening one arm (by half), would change the task. This pair, however, is no longer isoenergy, with larger energy response found for the $\triangle$. Thus, symmetric performances reported here could be caused by energy differences and/or texsize differences (the latter of which is now quite small). It should be noted that using blobs also changes energy variability across the orientation spectrum (as seen by the curves of Figure 1 at low spatial frequencies). In fact for Pairs 1.5 and 1.6 , both elements now have similar variability profiles and, thus, were not expected to be discrim- inable on the basis of energy variability as described by Rubenstein and Sagi (1990).

Until this point, it appears that texsize is an important property in this task, but is it the only one involved? Experiments 2 and 3 were designed to determine this more conclusively and establish the importance of line terminations.

\section{Experiment 2: Line Terminations Are Critical in O/C Task}

The design of this experiment was to determine the importance of line terminations in discriminating between two elements. Williams and Julesz (1991) conducted experiments with Pair 2.1 (see Figures $4 \mathrm{a}$ and 5) and demonstrated that discrimination between a $O$ (actually an octagon, but we use a circle symbol for convenience) and a $C$ (an octagon with a gap) was possible and that the $C$ was the more visible element. These elements were chosen because they are approximately isoluminous, with the $C$ having $12.5 \%$ fewer pixels. Barchilon Ben-Av and Sagi (1995) 


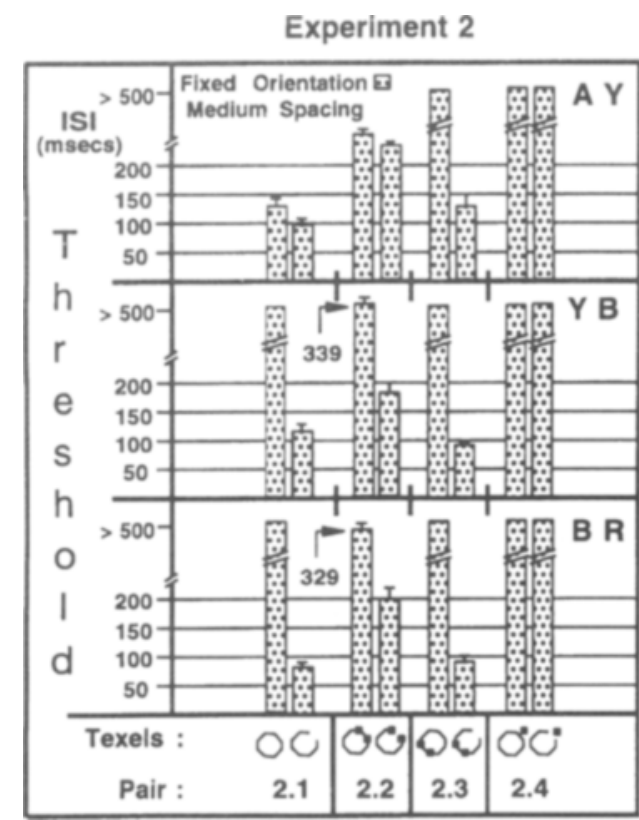

Figure 5. Psychophysical data of Experiment 2 are depicted for 3 observers and four different pairs of elements. Notice that, for most pairs (except Pair 2.4), the $C$ is the more visible foreground element. Comparing thresholds of $C$ foreground stimuli of Pairs 2.1 and 2.3 shows little difference. It is only when blobs are placed upon the line terminations (Pair 2.2) or in the near proximity (Pair 2.4) that performance suffers. For more details, see Figure 3.

showed that identical textures differing in brightness by such a small percentage (less than $20 \%$ ) are indiscriminable. Also, elements were of fixed orientation such that there was no energy variability across orientation (Williams \& Julesz, 1991), and, thus, discrimination could not be based on these types of energy gradients. In Experiment 2 , four pairs were created, adding square blobs to each pair in identical locations, so as to determine which part of the element was critical to the task. As seen in Pair 1.8 of Experiment 1, discrimination was impossible for peripheral targets. Therefore, similar to the stimuli used by Williams and Julesz (1991), a near-foveal foreground, mediumspacing stimulus was used (see Figure 4) to make the task easier. Elements were scaled such that each element was 60 pixels wide.

Data are depicted in Figure 5. For Pairs 2.1 and 2.3, the degree of performance asymmetry is quite similar, with the $C$ always being the more visible foreground relative to the $O$ foreground stimulus, which, in many cases, gives rise to an extremely difficult task. Note that because the blobs of Pair 2.3 caused relatively little change in performance relative to that of Pair 2.1, it appears that, although the blobs are quite substantial in size and brightness, they can be essentially ignored by the visual system when placed in noncritical locations (i.e., not near the line terminations). For Pair 2.2, with the line terminations covered (or blotted out), thresholds for the $\mathrm{C}$ foreground increased significantly relative to those of Pairs 2.1 and 2.3 [ from 99 to $197 \mathrm{msec}$, $p<.005, t$ test comparing observers' average of $2.2(n=3)$ with that of 2.1 and $2.3(n=6)$ ], indicating that some critical stimulus property (i.e., line termination) had been masked. Also, thresholds dropped for the $\mathrm{O}$ foreground stimulus relative to those of Pair 2.3 [from 500 to $305 \mathrm{msec}$, $p<.005, t$ test comparing observers' average of $2.2(n=3)$ with that of $2.3(n=3)$ ], indicating that the corresponding $C$ in the background was not as "noisy" or did not produce as many spurious (feature) gradients that can reduce foreground visibility. For Pair 2.4, discrimination was nearly impossible, indicating that the placement of the square blobs was also at a critical location. Note that, for Pair 2.2, some discrimination was possible, indicating that perhaps there was some other feature involved. Computer modeling with linear filtering of the elements showed that the $\mathrm{C}$ does in fact have a small orientation feature $\left(45^{\circ}\right.$ white blob sticking into the element through its gap), but data of Pair 2.4 show that filling this gap with a square blob makes the task impossible. Incidentally, the blobs of Pair 2.2 were positioned on the line terminations in such a way that the gap size remained unchanged. This ensured that differences in the data between Pairs 2.1 and 2.2 would not be gap-size dependent.

\section{Experiment 3: Line-Termination-Based Segmentation Is Not a Preattentive Process}

Thus far, Experiment 1 has shown that texsize of a texture is an important property in the $\Delta / \mathrm{L}$ task and that line terminations are critical for the $\mathrm{O} / \mathrm{C}$ task. But, unfortunately, a contradiction underlies these results: if line terminations are important, then all pairs of Experiment 1 would be discriminable, independent of the texsize. The first part of the solution to this contradiction is provided by the data of Pair 1.8, showing that peripheral discrimination is not possible with line terminations as the critical stimulus property. Thus, line terminations are not detected so easily. Treisman (1985) has shown that the O/C pair can be discriminated in parallel across the visual field, implying a "preattentive" process. However, it is still possible that distributed attention is being used in the task (Treisman \& Gormican, 1988), since parallel processes may also be attentive (Adini \& Sagi, 1992). The purpose of Experiment 3 was to determine whether this stimulus property is extracted preattentively or whether it requires attentive resources. This was accomplished by using a double-task experiment (Braun \& Sagi, 1990). Using the same stimulus design as that for Experiment 2 (a near-foveal foreground, medium-spacing stimulus), an additional task was placed in the center (see Figure 4a) and performance was recorded for three different pairs. Scaling to interelement ratios was maintained from previous experiments, with Pair 3.3 identical to Pair 2.1 (see Figure 6). Pair 3.1 was similar to Pair 1.1 with random orientations, except it was scaled to fit within a $39 \times 39$ pixel space so as to match the ratio (element scale to interelement distance) of Experiment 1. Pair 3.2 was similarly scaled and randomly oriented.

Data are depicted in Figure 6, with thresholds presented for stimuli with and without the additional central task. For Pairs 3.1 and 3.3, single-task data were identical to those of Experiments 1 and 2, respectively. For Pair 3.2, single- 
Experiment 3

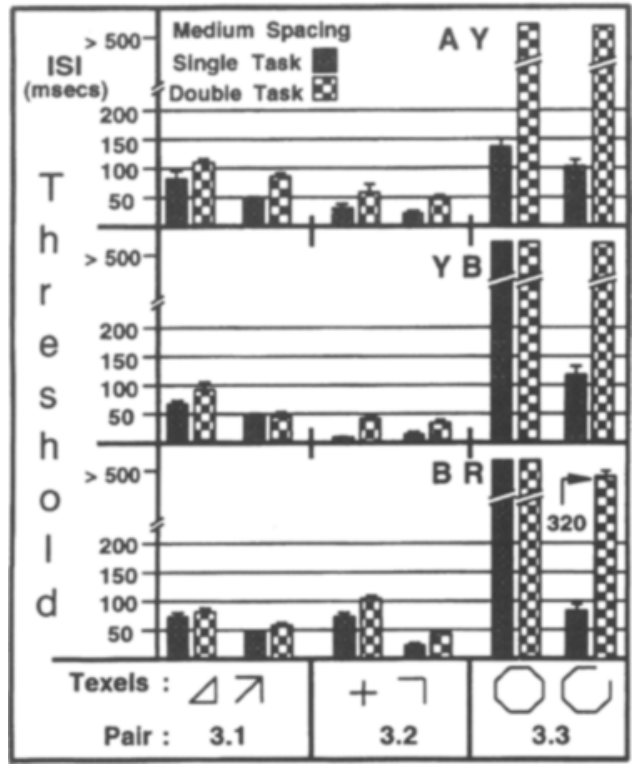

Figure 6. Psychophysical data of Experiment 3 are depicted for 3 observers and three different pairs of elements. Each stimulus was performed as a single task (peppered rectangles) or in conjunction with another difficult foveal task (checkerboard rectangles). Notice that, for Pairs 3.1 and 3.2, introducing the second attentive task has very little effect on thresholds (compare adjacent rectangles), whereas, for Pair 3.3, a large increase in threshold is shown for the $C$ foreground stimulus. This supports the notion that line terminations cannot be extracted at the preattentive level.

task thresholds were as expected, with the $L$ as the more visible of the pair (see Gurnsey \& Browse, 1987, for comparison). For double tasks, thresholds increased somewhat (21.3 msec, averaged across all data for Pairs 3.1 and 3.2) relative to thresholds for the single task for Pairs 3.1 and 3.2. Though this increase is statistically significant $(p<$ .0005 , pairwise $t$ test with 3 observers $\times 4$ conditions, total 12 paired measurements), a much larger increase was observed for Pair 3.3 [319.2 msec, $p<.005$, pairwise $t$ test with 4 paired measurements (out of 6 measurements of Pair 3.3 since, in two cases, saturation did not allow for comparison)]. (Note that comparisons between single- and double-task conditions for each observer for Pairs 3.1 and 3.2 yield statistical significance for only 7 out of 12 , whereas all nonsaturated comparisons for Pair 3.3 are significant.) This latter result indicates that the $\mathrm{O} / \mathrm{C}$ task is an attentive task, with line terminations that can only be extracted by using attention. Finally, because performance of the $\triangle / K$ task did not suffer from the central task, line terminations appear to have no bearing on this task, despite only one element having them ( $\angle$ has three line terminations). It should be noted that originally we tried to explicitly blot out the line terminations of the $K$ (while also placing blobs in the same locations on the $\Delta$ ); however, because these elements no longer had iso-second-order statistics, the resultant discrimination might have only been related to energy differences. Also, a slight decrease in performance for Pairs 3.1 and 3.2 with the introduction of an additional task is not unexpected, as the load on memory increases (now there are two responses to be remembered during each trial).

\section{SCALING AND INTERELEMENT DISTANCE: A PARADIGM FOR DETERMINING CRITICALITY OF TEXSIZE}

\section{Experiment 4: Texsize Changes With Scaling}

Since texsize has been established as a critical property in the $\Delta / K$ task, the present experiments will attempt to explore how other parameters of a texture stimulus, such as element scale and interelement distance, can affect the texsize and thus limit performance. The experiments conducted were motivated by psychophysical data of Gurnsey and Browse (1989), which showed that, for circle elements differing in size (or diameter), discrimination was possible, and the larger element was more visible when in the foreground. They also demonstrated that by increasing the size difference (they used six pairs of elements differing in size from $9 \%$ to $38 \%$ ), performance increased for both stimulus types (i.e., each element in turn was in the foreground) as did the performance asymmetry. Our Experiment 4 attempted to understand how this performance asymmetry is affected by altering the scale of the elements, while interelement distance remains fixed. Since texsize is jointly dependent on a texture element's size and the interelement spacing, it was of interest to determine whether texsize was a better indicator of performance asymmetry than size, as defined by Gurnsey and Browse. Gurnsey and Browse used stimuli whose interelement distance was 2.5 times the diameter of the circle. Here, we used circles that differed in size by $25 \%$ and whose scale ranged from small to big enough to be almost touching the adjacent elements. The peripheral foreground, small-spacing (64 pixels) stimulus was used (see Figures $7 \mathrm{a}$ and $7 \mathrm{~b}$ ), with the smaller of the circles scaled to fit within a square space of 15,25 , 35 , and 44 pixels on a side (see Figure 8, for more details). Photometric measurements of elements scaled to fit within a $25 \times 25$ pixel space produced luminances of $2.2 \mathrm{~cd} / \mathrm{m}^{2}$ and $2.3 \mathrm{~cd} / \mathrm{m}^{2}$. This $4 \%$ difference in luminance has been shown to be indiscriminable for textures (Barchilon BenAv \& Sagi, 1995); thus, these elements are perceptually isoluminous within this paradigm. Elements were of fixed orientation.

Data are depicted in Figure 9a. For the two smallest scaled pairs (Pairs 4.1 and 4.2), psychophysical results were similar to those of Gurnsey and Browse (1989), with lower thresholds for the larger circle (defined by the width) of the pair. For Pair 4.3, there was evidence of unstable behavior, with performance asymmetry remaining the same for 2 observers but reversing for the third (A.Y.). Finally, the largest scaled circles produced reversed asymmetry, with the smallest circle of the pair being more visible. The data indicate that interelement distance, relative to the size of the elements, is certainly a critical parameter, particularly for tasks with size as a critical property.

This behavior may be understood by observing that, as the large scaled elements begin to situate themselves close 

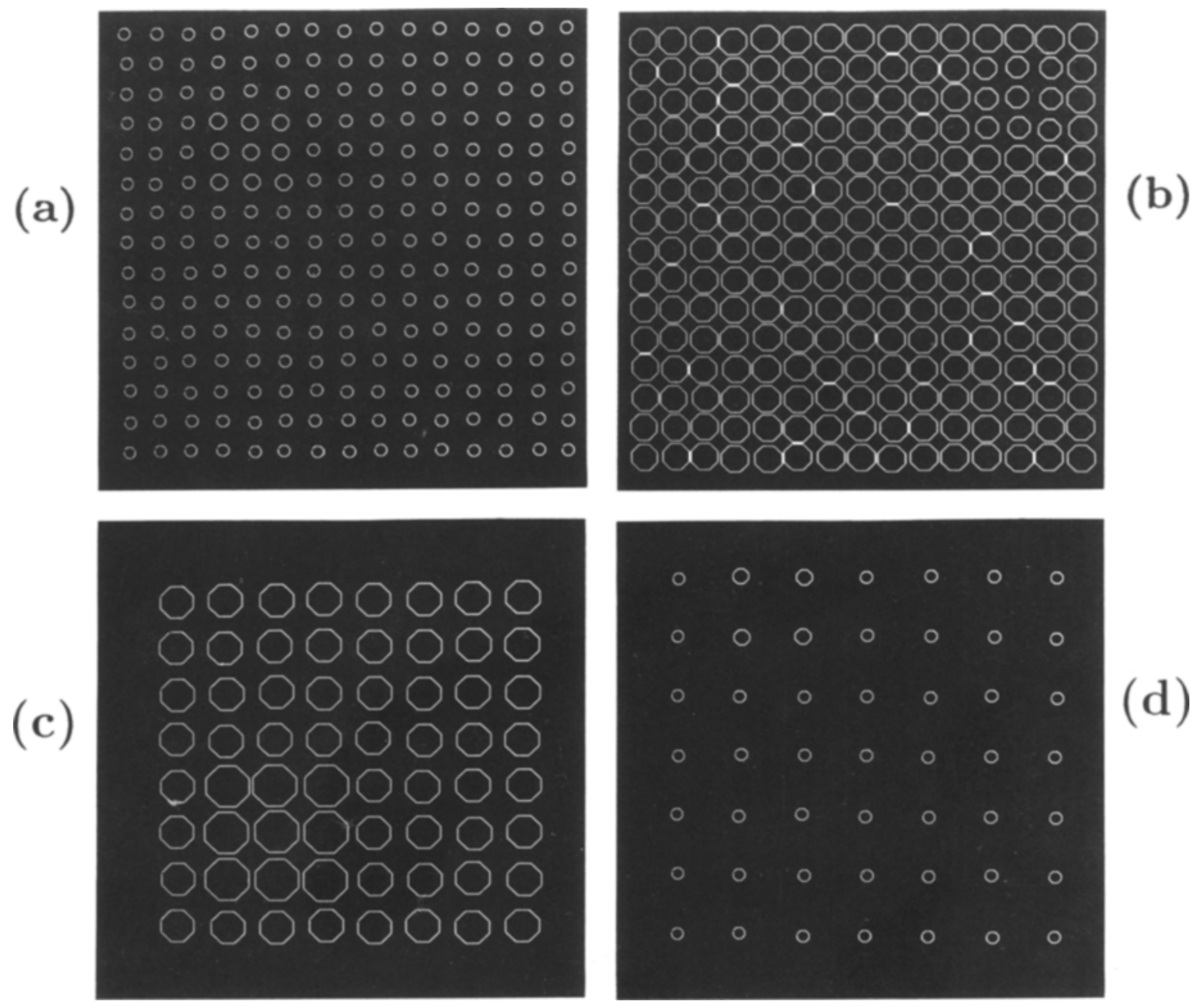

Figure 7. Sample stimuli used in Experiments 4, 5, and 6. Experiment 4 used a peripheral foreground, small-spacing stimulus (panels a and b), Experiment 5 used a near-foveal foreground, medium-spacing stimulus (panel c), and Experiment 6 used a peripheral foreground, large-spacing stimulus (panel d). All experiments used elements of different scales (compare panels a and b).

to their neighbors, their physical (or spatial) independence diminishes to the extent that they are as one with the adjacent elements. It is almost as if the larger circle of Pair 4.4 has become part of a mesh (i.e., no longer a set of large elements, but a finer entanglement of lines with little space between elements), while the smaller circles remain independent and, thus, their size remains intact (see Figure 7b). Filter analysis of these elements showed that the response weighted average of the filter wavelength, $\bar{\lambda}$, corresponded very closely to the above explanation. In particular, $\bar{\lambda}$ s (with larger wavelengths synonymous with a larger texsize) for elements of Pairs 4.1 and 4.2 indicate that the larger circle texture has the larger texsize, whereas for Pairs 4.3 and 4.4, the smaller circle texture has the larger texsize (see Figure 8, for filter analysis). An alternative explanation for the asymmetry reversal could be based on illusory interelement objects that may begin to play a more important role in the task as the scale of the elements increases and elements begin to become close to each other. At large scales, the smaller of the elements have larger illusory objects and, thus, performance asymmetry could be caused by size differences based on interelement spaces. A question with this explanation would be how to determine when the role of interelement spaces begins to dominate over the elements themselves (as scale increases). Also, it should be mentioned that when $\bar{\lambda}$ s are the same for both elements (like for Pairs 1.5 and 1.6), this does not always ensure

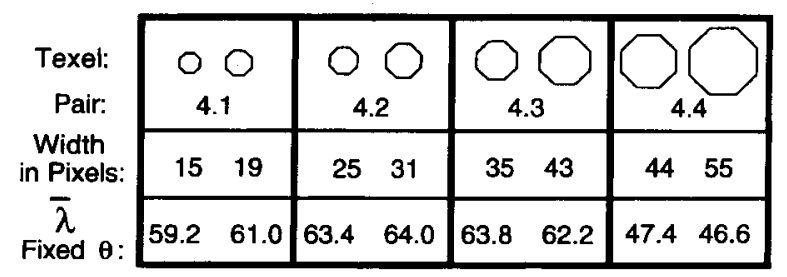

Figure 8. Pairs of elements used in Experiment 4. Below each element is its width and $\bar{\lambda}$ (both expressed in pixels). 


\section{Experiment 4}

Experiment 5

Experiment 6

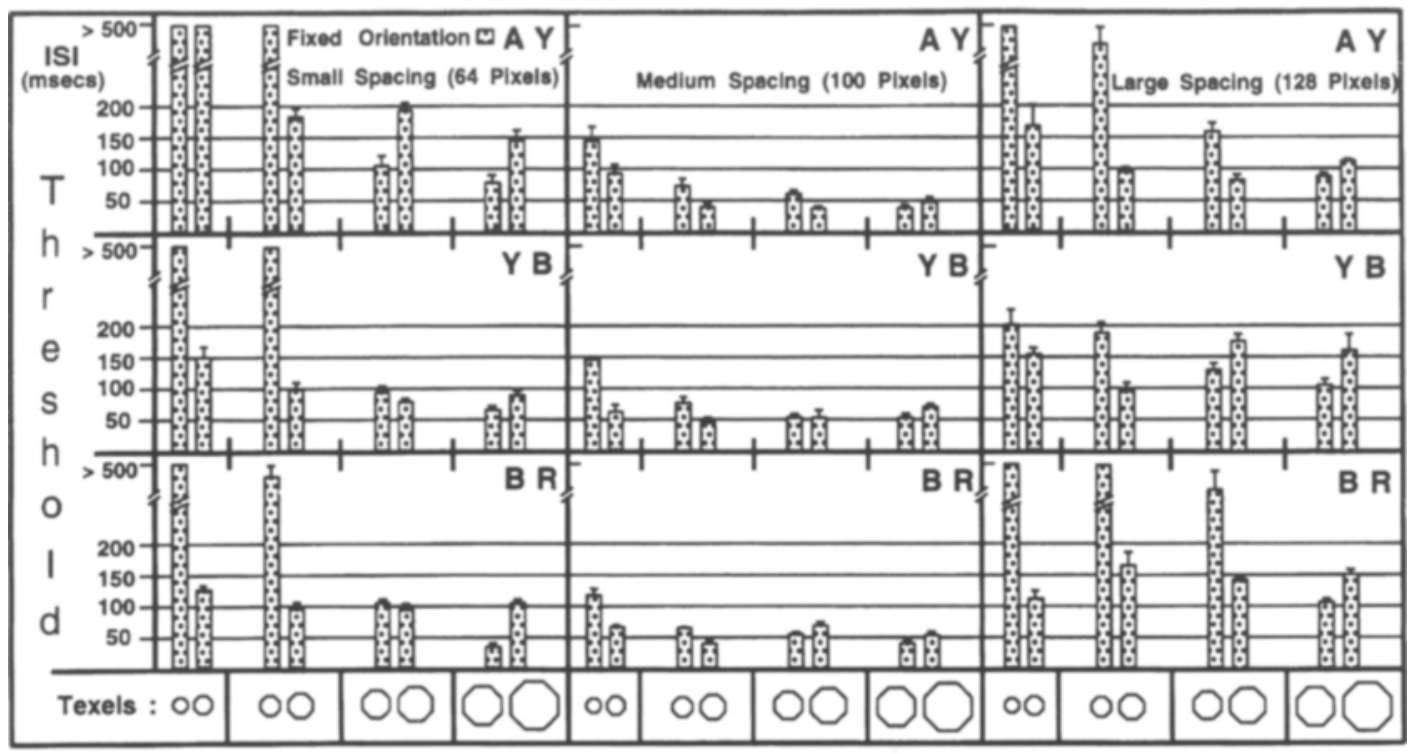

(a)

(b)

(c)

Figure 9. Psychophysical data of Experiments 4, 5, and 6 are depicted for 3 observers and four different scalings of the same pair of elements. Each experiment used a different interelement distance: Experiment 4 used 64 pixels (panel a), Experiment 5 used 100 pixels (panel b), and Experiment 6 used 128 pixels (panel c). In each experiment, elements were scaled in such a way as to preserve the ratio of element width (in pixels) to the interelement distance. Data from all experiments show that, for small element scales (Pairs 4.1 and 4.2), the larger of the circles (defined by the width) is more visible when in the foreground. However, for larger scales (Pairs 4.3 and 4.4), this performance asymmetry reverses, with the smaller of the circles now more visible. This implies that interelement distance plays a role in texture discrimination tasks, but changing the visual angle of the stimulus does not.

poor discriminability. Particularly, because the elements of Experiment 4 have different widths, they no longer are isoenergy and thus discrimination will always be possible.

\section{Experiments 5 and 6: Asymmetry Is Independent of Varying Interelement Spacing}

The results of the above experiments showed how element scale, relative to interelement distance, can alter the texsize of a texture. In these experiments, the interelement distance was altered to determine if the same behavior remained. This parameter is of interest because it is uncertain whether performance with stimuli scaled to different visual angles would produce different results. Accordingly, Experiment 4 was repeated two more times, but for the other stimulus types described in the Stimulus Design and Procedure section. Element scales were chosen so that the ratio between the elements' width (or diameter) and the interelement distance remained identical to those used in the previous experiment. For Experiment 5, a near-foveal foreground, medium-spacing (100 pixels) stimulus was used (Figure 7c), with the smaller circles of the pairs scaled to fit within a square space of $20,39,46$, and 68 pixels on a side, and $24,49,57$, and 85 pixels, respectively, for the larger circles. For Experiment 6, a peripheral foreground, large-spacing (128 pixels) stimulus was used (Figure 7d), with the smaller circles of the pairs scaled to fit within a square space of $25,50,58$, and 87 pixels on a side, and 31 , 63,73 , and 109 pixels, respectively, for the larger circles.
Data are depicted in Figures $9 b$ and $9 c$. The data indicate that the absolute interelement distance had little bearing on this size/spacing ratio-dependent behavior, because performance asymmetry flipped as the element scale increased for all three experiments (Experiments 4, 5, and 6). Inspection of individual thresholds of Experiment 5 indicated an easier task; however, this appears to be a result of foregrounds positioned near the fovea. For isoeccentric foregrounds of Experiments 4 and 6, thresholds appear to be approximately the same, indicating a visual system that is invariant to the visual angle for these stimuli.

\section{Experiments 7 and 8: Criticality of Texsize}

for $\triangle I K$ and $+/ L$ Tasks

Experiment 4 was again repeated, but this time for the $\Delta / L$ and $+/ L$ pairs. These experiments were conducted to determine whether behavior for stimuli composed of circle elements could be repeated for other pairs. According to Experiments 1, 2, and 3, texsize is an important stimulus property of the $\Delta / K$ task; hence, a flip in performance asymmetry using this scaling paradigm would be expected. In Experiment 7, a near-foveal foreground, medium-spacing stimulus was used for this pair with elements scaled to fit within a square space of $20,39,46$, and 66 pixels on a side (see Figures 10a, 10b, and 11). In Experiment 8 , for the $+/ L$ pair, a peripheral foreground, smallspacing stimulus was used, with elements scaled to fit within a square space of $15,25,29$, and 39 pixels on a side 
(see Figures 10c, 10d, and 11). A peripheral foreground was used here because this latter pair has been shown to be an easily discriminable pair relative to the $\Delta / K$ pair (Gurnsey \& Browse, 1987), and, subsequently, pilot studies with the near-foveal foreground proved too easy for the $+/ L$ task (many thresholds approached $0 \mathrm{msec}$ ). Both experiments used randomly oriented elements and elements of fixed orientation.

Data are depicted in Figures 12 and 13. For the $\triangle / K$, data were similar to those of Experiment 4 , indicating again that texsize is an important stimulus property of the task. This performance asymmetry reversal is also independent of fixed or randomly oriented elements, indicating that elements that are no longer isoenergy (fixed orientation) have no affect on the task. Similarly, filter analysis indicated a reversal of the larger $\bar{\lambda}$ element (between pair elements), such that this larger $\bar{\lambda}$ element was always more visible (see Figure 11). For $+/ L$ pairs of different scales, the $L$ always remained more visible when in the foreground (for Pair 8.1 of A.Y., the scale apparently was too small to do the task). Filter analysis also supports this behavior, with the $L$ always having a larger $\bar{\lambda}$. Rubenstein and Sagi (1990) also performed experiments with the $+/ L$ pair and found similar results for elements of random orientation. For stimuli with densely packed elements of fixed orientation (22\% more than Pair 8.4), they found a performance symmetry. These data may be explained by results from Pair 8.4 showing two cases of symmetry (A.Y. and B.R.). With more sparsely packed elements of fixed orientation, similar to Pair 8.2, they found some indication of symmetry but also one case of asymmetry (like that of the data of Pair 8.2). The data show that this scaling paradigm indicates criticality of texsize as a stimulus property, with more evidence that texsize is important in the $\Delta / K$ task, but less for the $+/ L$ task.

\section{Experiments 9 and 10: Altering Scale of One Element Affects Task; Orientation Variability Is Still Critical}

To test the relative strength of texsize as a stimulus property compared with energy variability across the orientation spectrum, Experiments 7 and 8 were performed again,
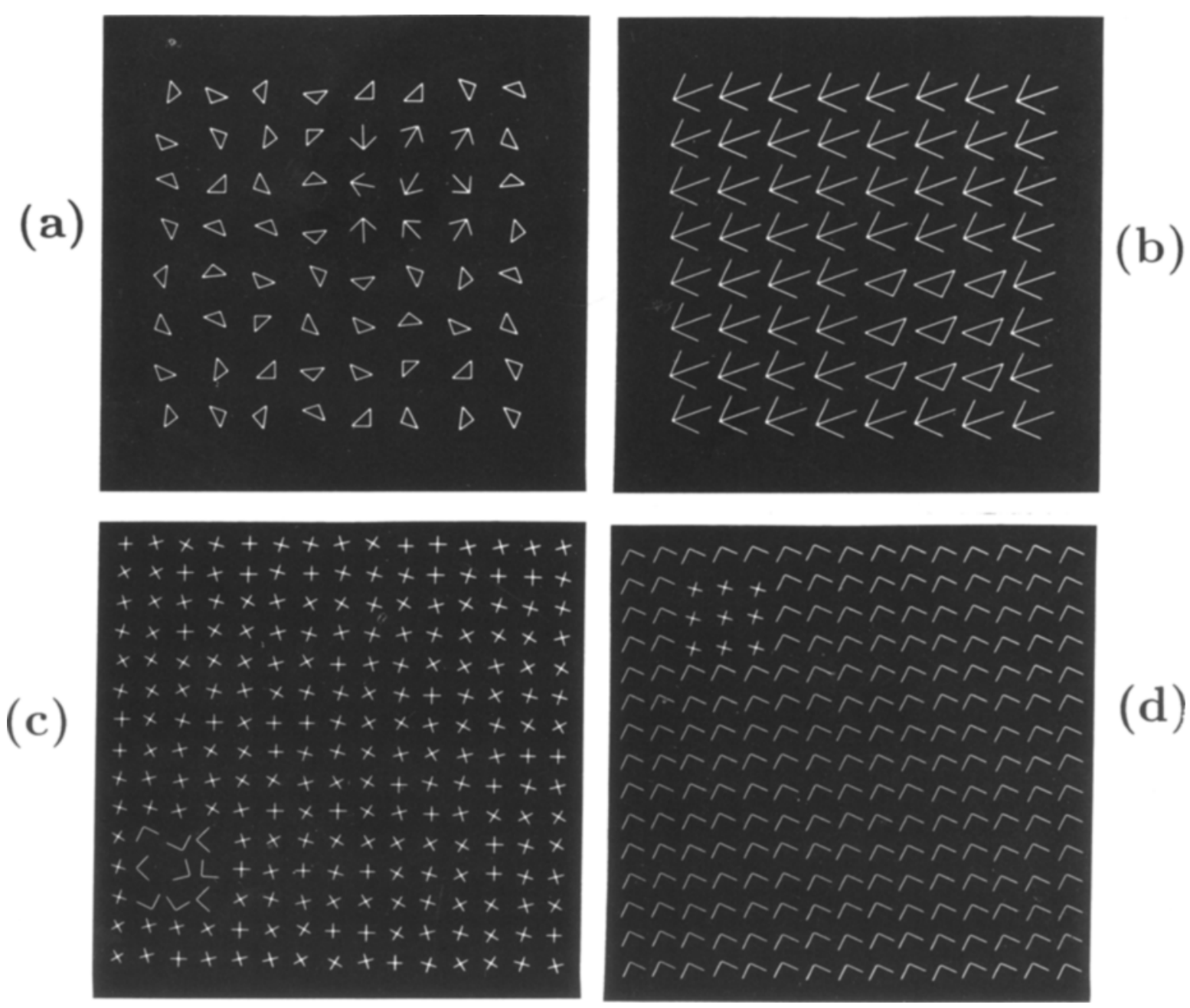

Figure 10. Sample stimuli used in Experiments 7 and 8. Experiment 7 used a near-foveal foreground, medium-spacing stimulus (panels a and b), and Experiment 8 used a peripheral foreground, small-spacing stimulus (panels $c$ and d). Both fixed-and random-orientation formats were used, as were different scales of the elements (compare panels a and $b$ ). 


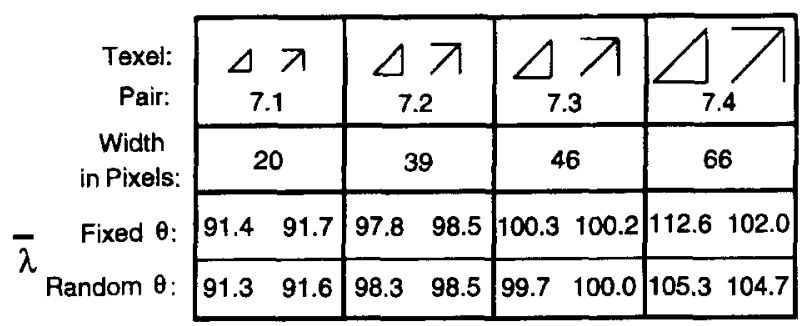

\begin{tabular}{|c|c|c|c|c|}
\hline $\begin{array}{l}\text { Texel: } \\
\text { Pair: }\end{array}$ & +7 & +7 & $\begin{array}{l}T_{8.3} \\
\end{array}$ & $T_{8.4}$ \\
\hline Width & 15 & 25 & 29 & 39 \\
\hline & $56.4 \quad 58.5$ & $60.0 \quad 62.9$ & $60.5 \quad 62.8$ & 62.678 .6 \\
\hline${ }^{\wedge}$ Random $\theta$ : & \begin{tabular}{|ll}
57.4 & 58.8
\end{tabular} & $59.8 \quad 62.8$ & 60.6 & $62.4 \quad 66.4$ \\
\hline
\end{tabular}

Figure 11. Pairs of elements used in Experiments 7 and 8. Below each element is its width and $\bar{\lambda}$ (both expressed in pixels). Filter analysis was done for both fixed-and random-orientation elements.

except this time by fixing the scale of one element and then changing the scale of the other. Hence, when the texsize $(\bar{\lambda})$ of the textures are the same, performance symmetry would indicate a texsize-influenced task, and performance asymmetry would imply that some other stimulus property dominates. Thus, in Experiment 9, for the $\Delta / K$ pair, a near-foveal foreground, medium-spacing stimulus was used, with the $K$ scale fixed to fit within a $20 \times 20$ pixel space and the $\Delta$ scaled to fit within a square space of $20,27,39$, and 60 pixels on a side (see Figures 14a, 14b, and 15). In Experiment 10, for the $+/ L$ pair, a peripheral foreground, small-spacing stimulus was used, with the $L$ scale fixed to fit within a $15 \times 15$ pixel space and the + scaled to fit within a square space of $15,25,29$, and 39 pixels on a side (see Figures 14c, 14d, and 15). All elements were randomly oriented.

Data are depicted in Figure 16. As one element increased in scale ( $\triangle$ and + ), it became the more visible of the pair. For the $\Delta / K$ data set, A.Y. and Y.B. showed signs that the task had become too easy for Pairs 9.3 and 9.4; however, data for Pair 9.2 already showed that performance asymmetry had flipped. Filter analysis (see Figure 15) also showed this asymmetry reversal between Pairs 9.1 and 9.2. Data for the $+/ L$ pair are similar to those of Bergen and Adelson (1988), who also demonstrated that by decreasing the scale of only one of the elements (the $L$ element), performance decreases (we instead increased the scale of the + , but there is no real psychophysical difference). The most important effect relates to comparison of thresholds of Pairs 9.1 and 9.2 and comparison of thresholds of Pairs 10.1 and 10.2. Between these pairs is the critical point at which the larger $\bar{\lambda}$ element of the pair changes. At this point for the $\Delta / K$ pair, reversal of performance asymmetry was observed, indicating that for this task texsize is the dominant property. However, for the $+/ L$ pair, performance asymmetry did not reverse and the
$L$ continued to have lower thresholds until the + became very large (Pair 10.3 for Y.B. and Pair 10.4 for A.Y. and B.R.). This indicates that, despite the + texture having the larger texsize in Pair 10.2 (Figure 15), it appears that some other feature is affecting the task. Rubenstein and Sagi (1990) showed that, in analyzing energies for different filters, the $L$ has much more energy variability across the orientation spectrum than does the + , and they claimed this variability in the background can limit performance. Thus, it appears for Pair 10.2 that this variability is still dominant and gives rise to large thresholds for the + foreground stimuli. It is not until the + has become significantly larger than the $L$ (e.g., Pair 10.4) that differences in texsize can dominate over any effect caused by energy variability across orientation. It should be noted that the $\Delta$ has been shown to have more energy variability across orientation than does the $K$ (see Figure 1); however, because it is relatively small (relative to the $L$ ), its contribution to the task is negligible.

\section{DISCUSSION}

The main objectives of this work were to demonstrate that, within the framework of visual textures, the texsize of microelements (see the Appendix for definition) in a texture is a critical property in preattentive tasks and that line terminations have high saliency, but only within the attentive framework. Gurnsey and Browse (1989) demon-

\section{Experiment 7}

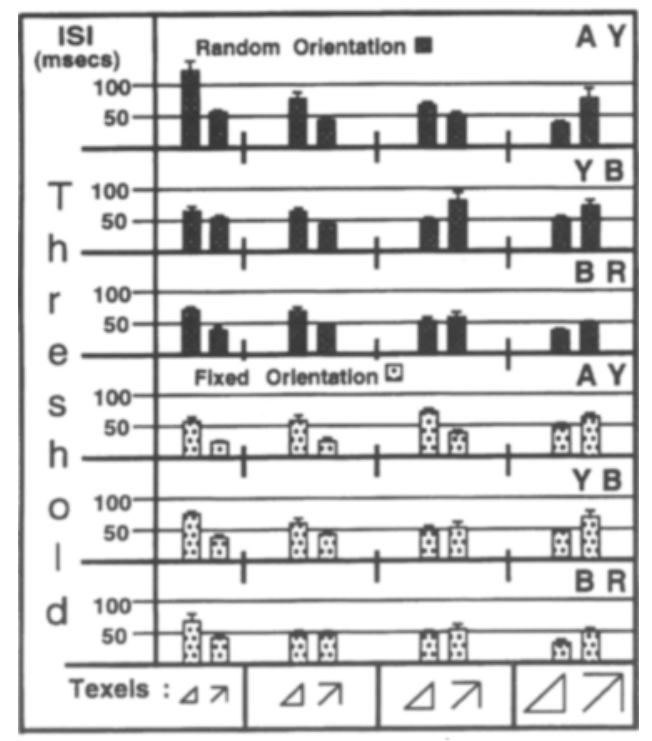

Figure 12. Psychophysical data of Experiment 7 are depicted for 3 observers and four different scalings. Elements were presented either in a fixed-orientation (light rectangles) or a randomorientation (dark rectangles) format. Data of both stimulus types show that, for small element scales (Pairs 7.1-7.3), the $K$ is more visible when in the foreground. However, for the largest scale (Pair 7.4), this performance asymmetry reverses, with the $\Delta$ now more visible. This asymmetry reversal correlates well with texsize (see Figure 11) and implies that texsize is a critical property in the task. 


\section{Experiment 8}

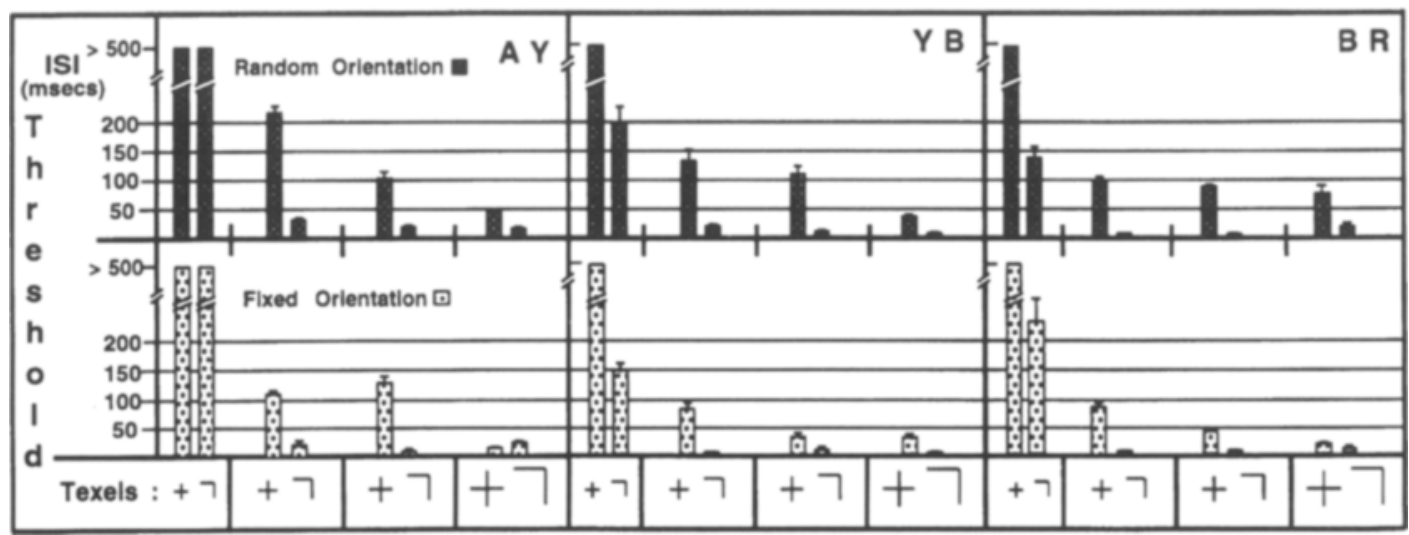

Figure 13. Psychophysical data of Experiment 8. Data indicate that scaling elements have little effect on the basic performance asymmetry (i.e., $L$ is more visible in the foreground).

(a)
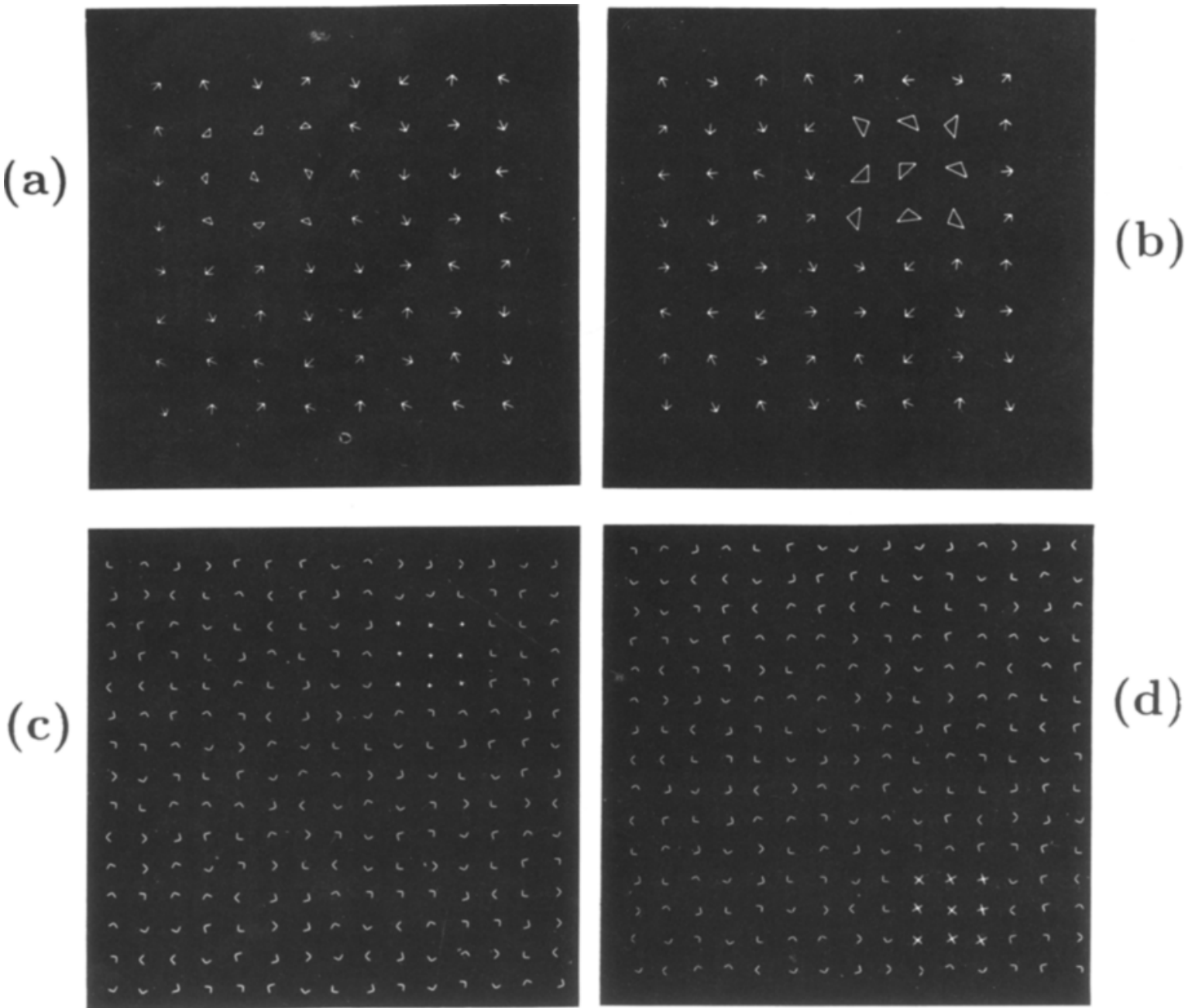

Figure 14. Sample stimuli used in Experiments 9 and 10. For both experiments, one of the elements had a constant scale $(\angle$ and $L$ ) and the other had one from a selection of different scales. Thus, according to the choice of scales, the $\triangle$ and + textures could have a smaller texsize, relative to the other pair element (panels a and c), or a larger texsize (panels b and d). 


\begin{tabular}{|c|c|c|c|c|}
\hline $\begin{array}{l}\text { Texel: } \\
\text { Pair: }\end{array}$ & $\begin{array}{c}\Delta \lambda \\
9.1\end{array}$ & $\begin{array}{c}\triangle \nearrow \\
9.2\end{array}$ & $\triangle_{9.3} \lambda$ & $\varlimsup_{9.4} \lambda$ \\
\hline in Pixels: & $20 \quad 20$ & $27 \quad 20$ & $39 \quad 20$ & $60 \quad 20$ \\
\hline$\stackrel{\lambda}{\text { Random } \theta:}$ & $91.3 \quad 91.6$ & $93.8 \quad 91.6$ & $98.3 \quad 91.6$ & $93.8 \quad 91.6$ \\
\hline $\begin{array}{c}\text { Texel: } \\
\text { Pair: }\end{array}$ & $\begin{array}{r}+7 \\
10.1 \\
\end{array}$ & $\begin{array}{c}+7 \\
10.2 \\
\end{array}$ & $\begin{array}{l}+7 \\
10.3\end{array}$ & $\left.T_{10.4}\right]$ \\
\hline in Pixels: & $15 \quad 15$ & $25 \quad 15$ & $29 \quad 15$ & $39 \quad 15$ \\
\hline$\underset{\text { Random } \theta:}{\lambda}$ & $57.4 \quad 58.8$ & $59.8 \quad 58.8$ & $60.6 \quad 58.8$ & $62.4 \quad 58.8$ \\
\hline
\end{tabular}

Figure 15. Pairs of elements used in Experiments 9 and 10. Below each element is its width and $\bar{\lambda}$ (both expressed in pixels). Filter analysis was done for randomly oriented elements.

strated size effects with stimulus elements of different sizes (i.e., circles with different diameters). They showed that the larger element will always be more visible when in the foreground. Here, we add more evidence by showing that size effects can also be important in other tasks that involve elements that are isoenergy (the circles mentioned above are not) and in those that have a different number of line terminations (e.g., the $\Delta / K$ pair). The importance of size in the $\triangle / K$ task was determined by adding bright blobs to both elements at various places (while maintaining iso-second-order statistics) and then noting differences in psychophysical performance. In light of these experiments, it was determined that the critical parameter was not element size (which is actually difficult to estimate), but rather the spatial frequency content of a texture region. This measure of texsize was computed using a filter analysis (based on Gabor filters) and represents the response weighted average of the filter wavelength, $\bar{\lambda}$, for a group of neighboring elements $(3 \times 3)$. Results from this analysis showed that adding blobs in such a way to make elements embedded in textures isotexsize (Pairs 1.5 and 1.6) will render a task nearly impossible (for visual correlation, see Figure 17). Also, the element with a larger texsize appears to be the more visible of the two (i.e., Pairs 1.1-1.5, which use isoenergy elements and thus have no other critical properties that might contribute to the task). Note that, although small differences in texsize (down to about $1 \%$ ) have been used to account for the experimental results as compared with a much larger luminance difference threshold for textures reported by Barchilon Ben-Av and Sagi (1995), the visual system may simply have more sensitivity to texsize.

Experiment 9

Experiment 10

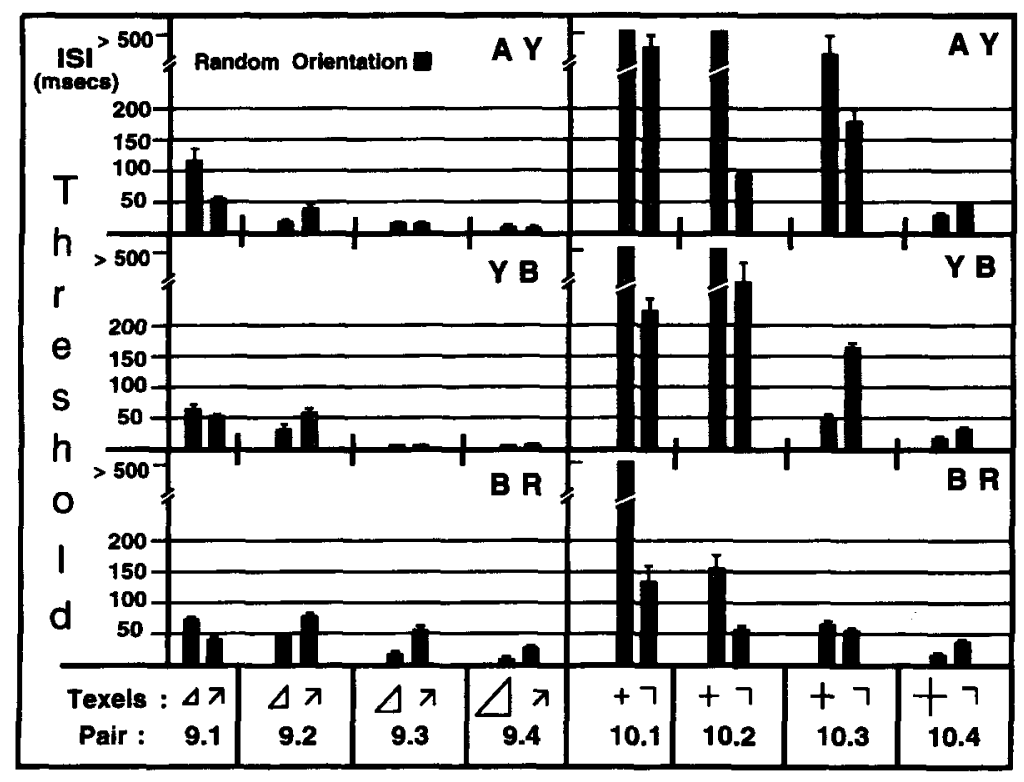

(a)

(b)

Figure 16. Psychophysical data of Experiments 9 and 10 are depicted for 3 observers and four different scalings. In each experiment, one of the element's scale ( $K$ and $L$ ) remained fixed, and the other became larger (defined by width in pixels). Data show that as the scaled element $(\angle$ and + ) increases in scale, it becomes the more visible of the pair and the performance asymmetry reverses. Notice, however, in Experiment 9 , that the asymmetry reversal occurs as soon as the $\angle$ texture has the larger texsize of the pair (Pair 9.2), whereas, in Experiment 10, the + texture must become significantly larger in texsize than that of the $L$ for the asymmetry to reverse (Pair 10.3 for Y.B., and Pair 10.4 for A.Y. and B.R.). This indicates the importance of filter-response variability across the orientation spectrum of the $L$, a stimulus property also critical to the task. 
Performance asymmetry based on texsize differences was also found to be influenced by the interelement distance. Experiments 4-6 established that larger circles (defined by the width) were not always more visible and that, as the scale of the elements increased and thus became closer to their neighbors, performance asymmetry reversed with the smaller of the elements becoming the pop-out. Filter analysis supported this psychophysical result, showing, for large element scales, that the smaller circle had the larger $\bar{\lambda}$ (see Figures 8 and 18). It is important to note that $\bar{\lambda}$ is based on inputting a $3 \times 3$ matrix of elements into the model. Thus, this measurement is no longer localized for each element, but rather for a slightly larger neighborhood. This result appeared to be independent of the absolute visual angle, since stimuli with different interelement distances were used producing similar results. Other element pairs $(\Delta / K$ and $+/ L)$ were also tested within this paradigm (Experiments 7 and 8 ), with a performance asymmetry reversal reported for the former pair (i.e., for small element scales, the $K$ was more visible, whereas, for larger scales, the $\Delta$ became the pop-out), and no reversal was found for the latter pair. Filter analysis of these elements at different scales also supported this result, with a larger $\bar{\lambda}$ corresponding to the more visible element. Note that, for the scale in which the $\triangle / K$ elements were approximately iso- $\bar{\lambda}$ (Pair 7.3), no discrimination would be predicted, as was the case for Pairs 1.5 and 1.6. However, further analysis

Filter Analysis Correlation to Psychophysics

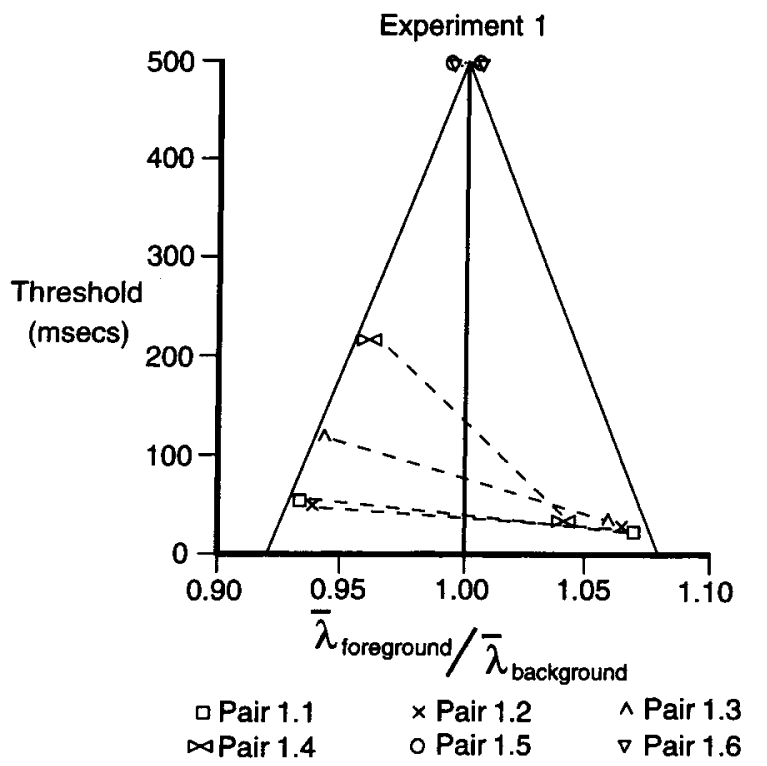

Figure 17. Correlation between filter analysis and psychophysics of isoenergy pairs of Experiment 1 depicts thresholds for each pair (with each element in the foreground) as compared with the ratio between $\bar{\lambda}$ each element of that pair. The ratio was computed by dividing data of the foreground element of the pair by that of the background element. Note that, in all cases, when the $\bar{\lambda}$ ratio is larger than one, the threshold is lower than the corresponding threshold when the $\bar{\lambda}$ ratio is less than one (corresponding points are connected by a dotted line). Also note that, when the $\bar{\lambda}$ ratio equals one, thresholds are very high (Pairs 1.5 and 2.6). See the Appendix for details. showed that large element scaling relative to the interelement distance can change the energy response. Particularly, at this scale, the $\Delta$ and the $K$ were found to have different energies, and thus discrimination would still be possible.

With these new results, we were also interested in determining how influential texsize can be in a task, relative to the response variability across the orientation spectrum (for randomly oriented elements), which has also been shown to be a critical stimulus property (Rubenstein \& Sagi, 1990). This was studied by repeating the aforementioned Experiments 7-8, except this time by fixing the scale of one of the elements $(K$ and $L)$, while allowing the other elements $(\Delta$ and + ) to change scales (Experiments 9 and 10). Computed $\bar{\lambda}$ values indicated a flip in the texsize between the elements of Pairs 9.1 and 9.2 and also the elements of Pairs 10.1 and 10.2 (see Figure 15); however, psychophysical results showed a performance asymmetry reversal only for the $\Delta / K$ pair, and not for the $+/ L$ pair. It was not until the + became much larger (in texsize) than the $L$ (Pair 10.4) that a reversal was observed (i.e., a more visible + ). Because the $L$ has been shown to have a large amount of response variability across the orientation spectrum relative to that of the + (Rubenstein \& Sagi, 1990), this offset asymmetry reversal could be caused by this property, which would maintain high thresholds for the + foreground stimuli (i.e., noisy gradients in the $L$ background). Thus, if the texsize, $\bar{\lambda}$, of a texture is larger, this does not ensure its greater visibility in the foreground, because spatial variability might still dominate the task.

All results reported so far indicate that texsize can be critical for a task and that a performance asymmetry exists, with the texture of the larger texsize as more visible. Unfortunately, until now, there has been little explanation for how this size asymmetry arises. Rubenstein and Sagi (1990) showed that asymmetry could be explained by signal detection theory, with a target's visibility limited by the "noise" in the background. They showed that this noise could be composed of spurious gradients in the background that acted to confuse the observer in his/her attempt to localize the background/foreground gradient. With the psychophysical results of Gurnsey and Browse (1987), they determined that one form of this noise could be filterresponse variability across the orientation spectrum (the stimuli had randomly oriented elements), which was then transformed into spatial variability. Within this framework, the larger texsize texture must somehow be "noisier," limiting the visibility of the smaller texsize texture when it is in the foreground. In other words, perhaps the low-spatialfrequency filters have more internal noise relative to that of the high-spatial-frequency filters. Hence, a large $\bar{\lambda}$ value would inherently imply filter activity of greater variability and would thus give rise to spatial variability in the system. An alternative theory was purported by Gurnsey and Browse (1989). This theory accounts for size-based performance asymmetry by using a Weber-like computation that normalizes the texture boundary by the overall activity of the entire image. This approach, in the context of texture segmentation, implies a global processing, which is not necessary considering the accumulating evidence 
Filter Analysis Correlation to Psychophysics

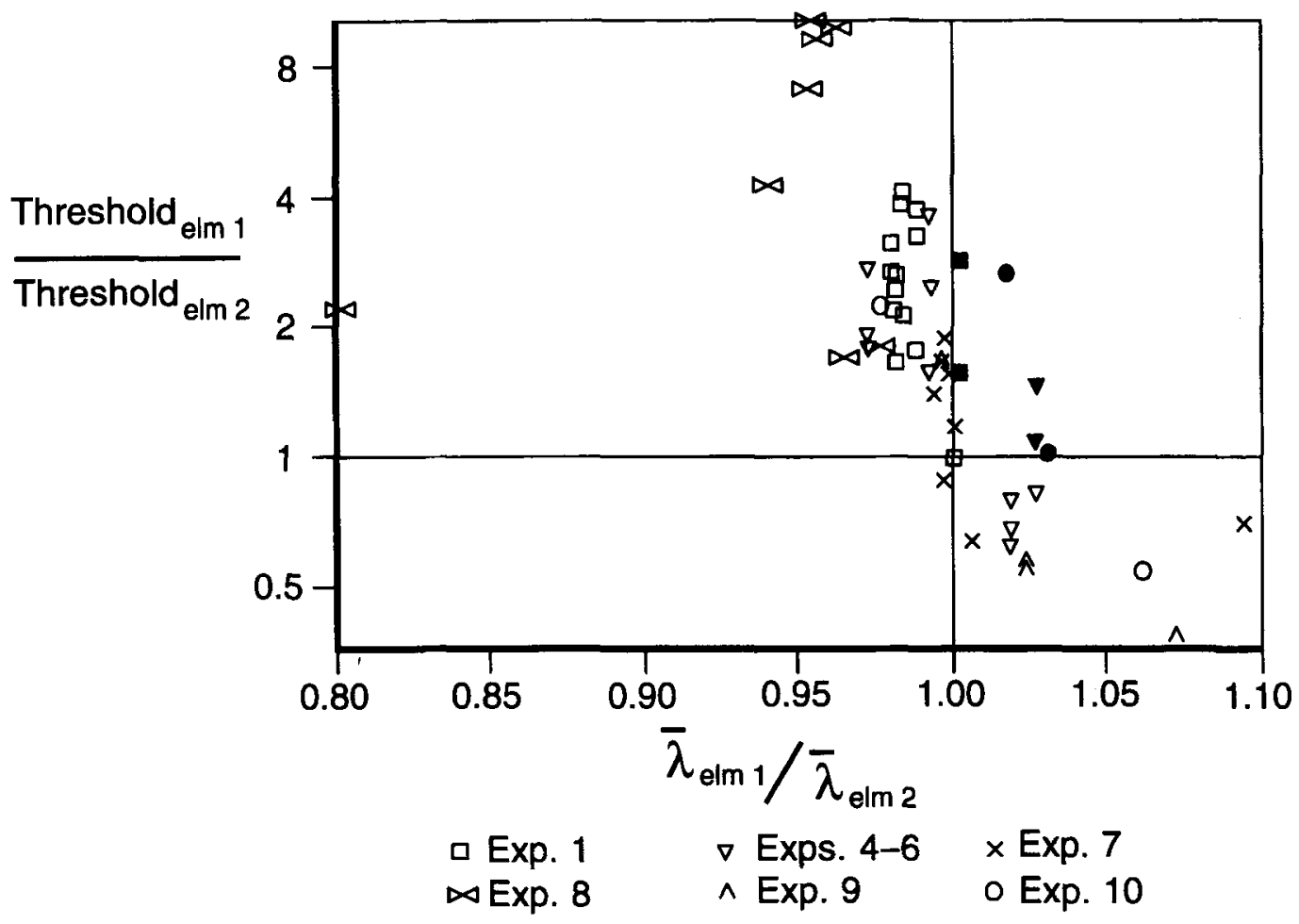

Figure 18. Correlation between filter analysis and psychophysics of all experiments examining the effect of texsize (Experiment 1 and Experiments 4-10). Depicted is the ratio of thresholds for each pair as compared with the ratio between $\bar{\lambda}$ for each element of that pair. Notice that most points fall within the top left or bottom right quadrants, indicating that the element with the larger $\bar{\lambda}$ is the more visible of the pair. All exceptions, shown as filled symbols, are explained in the Appendix.

that early vision and texture processes are local (Nothdurft, 1985; Sagi \& Julesz, 1987). On the other hand, the assumption on noise is consistent with local processing.

This proposed wavelength-dependent noise and the filter analysis described above have also been used to study other asymmetries reported in the literature. Particularly, Gurnsey and Browse $(1988,1989)$ showed that positionally jittered elements are more visible in the foreground (with identical but nonjittered elements in the background) than in the reverse case. Filter analysis of these elements showed that the jittered elements have a larger $\bar{\lambda}$, perhaps because these elements have a tendency to clump together and become a collection of larger blobs. Thus, this jitter asymmetry can be explained by texsize and wavelengthdependent internal noise. Treisman and Gormican (1988) showed that ellipses are more visible in the foreground amongst circles than in the reversed case (fixed orientation). Filter analysis indicated a larger $\bar{\lambda}$ for the ellipse, again rendering a task dependent on wavelength-dependent noise. Note that, if these elements were randomly oriented, orientation variability described by Rubenstein and Sagi (1990) would also come into play. Beck (1982) showed that longer lines are more visible in the foreground with shorter lines in the background (all with fixed orientation) than in the reverse case. Again, filter analysis indicated the longer lines in a texture as having a larger texsize.
The implications of the results presented here would necessitate an alteration to the Rubenstein and Sagi (1990) model, since, at present, it cannot account for performance asymmetry based on texsize differences. The proposed change to this model would include a modification to the internal noise, with more noise for the lower-spatialfrequency filters and less for the higher ones. Then, when all filtered maps are recombined in the latter stages, the element with the larger $\bar{\lambda}$ will give rise to more spurious gradients. We performed filter analysis on the 18 pairs used by Gurnsey and Browse (1987) to determine whether $\bar{\lambda}$ of these elements could solely account for the psychophysical results. For many cases, a larger $\bar{\lambda}$ correlated with the more visible element; however, for some pairs (7-12 of Gurnsey \& Browse, 1987), there were opposite predictions. This adds more evidence to the importance of orientation variability of these elements as the Rubenstein and Sagi (1990) model suggests.

An important result of this work pertains to understanding the influence of line terminations in texture tasks. Williams and Julesz (1991) reported a performance asymmetry when using stimuli composed of circles and partial circles (i.e., $C$ ), with discrimination possible for the $C$ foreground stimuli, although much less discrimination possible for the reversed case (elements were of fixed orientation, thus orientation variability could not be a factor). 
They explained this result within the context of subjective contours; however, here (in Experiment 2) we attempted to establish the importance of line terminations. Using similar stimuli, we added bright blobs to these elements at various places and found that, when blobs were placed on or in the near proximity of the line terminations of the $C$, discrimination was nearly impossible. This evidence supports the notion of line terminations as an important stimulus property. Experiment 3 attempted to determine whether this stimulus property was preattentively extracted (in parallel) or detected in an attentive (serial) fashion. This was determined by using a double-task paradigm developed by Braun and Sagi (1990). They showed that performance does not suffer in a preattentive task by introducing a second attentive task, although it does for attentive tasks. Hence, by using different pairs $(\triangle / K,+/ L$, and $O / C)$, we determined that line terminations $(\mathrm{O} / \mathrm{C})$ are in fact extracted at the attentive level, whereas performance of the other pairs suffered little from the additional difficult task.

\section{REFERENCES}

Adini, Y., \& SAGI, D. (1992). Parallel processes with the "spot-light" of attention. Spatial Vision, 6, 61-77.

Barchilon Ben-Av, M., \& SAGI, D. (1995). Perceptual grouping by similarity and proximity: Experimental results can be predicted by intensity autocorrelations. Vision Research, 35, 853-866.

BECK, J. (1973). Similarity grouping of curves. Perceptual \& Motor Skills, 36, 1331-1341.

BECK, J. (1982). Textural segmentation. In J. Beck (Ed.), Organization and representation in perception (pp. 285-317). Hillsdale, NJ: Erlbaum.

BECK, J., SUtTER, A., \& IVRY, R. (1987). Spatial frequency channels and perceptual grouping in texture segregation. Computer Vision, Graphics, \& Image Processing, 37, 299-325.

BERGEN, J. R., \& ADELSON, E. H. (1988). Early vision and texture perception. Nature, 333, 363-364.

Braun, J., \& SaGI, D. (1990). Vision outside the focus of attention. Perception \& Psychophysics, 48, 45-58.

CAELLI, T. M. (1985). Three processing characteristics of visual texture segmentation. Spatial Vision, 1, 19-30.

CAelli, T. M., \& Julesz, B. (1978). On perceptual analyzers underlying visual texture discrimination: Part I. Biological Cybernetics, 28, 167-175.

Caelli, T. M., Julesz, B., \& Gilbert, E. (1978). On perceptual analyzers underlying visual texture discrimination: Part II. Biological $\mathrm{Cy}$ bernetics, 29, 201-214.

Cannon, M. W., \& Fullenkamp, S. C. (1991). Spatial interactions in apparent contrast: Inhibitory effects among grating patterns of different spatial frequencies, spatial positions, and orientation. Vision Research, 31, 1985-1998.

Chubb, C., Sperling, G., \& Solomon, J. (1989). Texture interactions determine apparent lightness. Proceedings of the National Academy of Sciences, 86, 9631-9635.

DaUGMAN, J. (1985). Uncertainty relation for resolution in space, spatial frequency, and orientation optimized by two-dimensional visual cortical filters. Journal of the Optical Society of America A, 2, 1160-1169. FoGEL, I., \& S AGI, D. (1989). Gabor filters as texture discriminator. Biological Cybernetics, 61, 103-113.

GURNSEY, R., \& BRowSE, R. A. (1987). Micropattern properties and presentation conditions influencing visual texture discrimination. Perception \& Psychophysics, 41, 239-252.

Gurnsey, R., \& Browse, R. A. (1988). Aspects of visual texture discrimination. In Z. Pylyshyn (Ed.), Computational processes in human vision: An interdisciplinary perspective. Norwood, NJ: Canadian Institute for Advanced Research Series in Artificial Intelligence and Robotics.

GuRNSEY, R., \& Browse, R. A. (1989). Asymmetries in visual texture discrimination. Spatial Vision, 4, 31-44.

Hoffman, M. I., \& Hallett, P. E. (1993). Texture segregation based on two-dimensional relative phase differences in composite sine-wave grating patterns. Vision Research, 33, 221-234.

JuLESZ, B. (1981). Textons, the elements of texture perception, and their interactions. Nature, 290, 91-97.

JULESz, B. (1984). A brief outline of the texton theory of human vision. Trends in Neurosciences, 7, 41-45.

Julesz, B. (1986). Texton gradients: The texton theory revisited. Biological Cybernetics, 54, 245-251.

Julesz, B., Gilbert, E. N., Frisch, H. L., \& Shepp, L. A. (1973). Inability of humans to discriminate between visual textures that agree in second-order statistics. Biological Cybernetics, 31, 137-140.

Kovács, I., \& JulEsZ, B. (1993). A closed curve is much more than an incomplete one: Effect of closure in figure-ground segmentation. Proceedings of the National Academy of Sciences, 90, 7495-7497.

LANDY, M. S., \& BERGEN, J. R. (1991). Texture segregation and orientation gradient. Vision Research, 31, 679-691.

LEVITT, H. (1971). Transformed up-down methods in psychoacoustics. Journal of the Acoustical Society of America, 49, 467-477.

Malik, J., \& Perona, P. (1990). Preattentive texture discrimination with early visual mechanisms. Journal of the Optical Society of America A, 7, 923-932.

NeISSER, U. (1967). Cognitive psychology. Englewood Cliffs, NJ: Prentice-Hall.

NothdURFT, H. C. (1985). Sensitivity for structure gradient in texture discrimination task. Vision Research, 25, 1957-1968.

Rubenstein, B. S., \& SAGI, D. (1990). Spatial variability as a limiting factor in texture-discrimination tasks: Implications for performance asymmetries. Journal of the Optical Society of America A, 7, 1632-1643.

Rubenstein, B. S., \& SAGi, D. (1993). Effects of foreground scale in texture discrimination tasks: Performance is size, shape, and content specific. Spatial Vision, 7, 293-310.

SAGI, D., \& HochsTeIN, S. (1985). Lateral inhibition between spatially adjacent spatial-frequency channels? Perception \& Psychophysics, 37, 315-322.

SAGI, D., \& JULESZ, B. (1987). Short-range limitation on detection of feature differences. Spatial Vision, 2, 39-49.

Treisman, A. (1985). Preattentive processing in vision. Computer Vision, Graphics, \& Image Processing, 31, 156-177.

Treisman, A., \& GormiCAN, S. (1988). Feature analysis in early vision: Evidence from search asymmetries. Psychological Review, 95, 15-48.

Williams, D., \& JulEsz, B. (1991). Filters versus textons in human and machine texture discrimination. In H. Wechsler (Ed.), Neural networks for human and machine perception (pp. 145-175). New York: Academic Press. 


\section{APPENDIX \\ Estimation of Texsize Using Linear Filters}

The aim of this appendix is to develop a mathematical, quantitative analysis that correlates with human performance. Particularly, we would like to compute the apparent size for each element when in a texture or determine the response weighted average of the filter wavelength, $\bar{\lambda}$, for a group of neighboring elements. Gurnsey and Browse (1989) found that the larger of a stimulus's elements will always be more visible in the foreground; thus, if we can establish some correlation between this larger element (i.e., larger $\bar{\lambda}$ )and its greater visibility, then apparent size, or texsize, can be shown to be a critical stimulus property in the psychophysical task. The basis of this analysis is derived from the model of Rubenstein and Sagi (1990), which used linear filters to emulate the early visual system. They used Gabor filters that can be designed to be selective to a particular bandwidth of orientations and spatial frequencies. Using even and odd phase Gabor filters having fixed orientation and spatial frequency, we computed the filtered energy of various elements. The equations for these filters are:

$$
G_{e v}\left(x, y \mid \lambda, \theta, x_{c}, y_{c}\right)=e^{\frac{-\left(\left(x-x_{c}\right)^{2}+\left(y-y_{c}\right)^{2}\right)}{2 \sigma^{2}}} \cdot \cos \left\{\frac{2 \pi}{\lambda}\left(\left(x-x_{c}\right) \cos \theta-\left(y-y_{c}\right) \sin \theta\right)\right.
$$

and

$$
G_{o d}\left(x, y \mid \lambda, \theta, x_{c}, y_{c}\right)=e^{\frac{-\left(\left(x-x_{c}\right)^{2}+\left(y-y_{c}\right)^{2}\right)}{2 \sigma^{2}}} \cdot \sin \left\{\frac{2 \pi}{\lambda}\left(\left(x-x_{c}\right) \cos \theta-\left(y-y_{c}\right) \sin \theta\right)\right\},
$$

where $\sigma$ is the Gaussian width ( $\sigma=\lambda$, giving rise to a bandwidth of approximately 1.5 octaves), $\theta$ is the filter orientation, and $\lambda$ is its wavelength. $x_{c}, y_{c}$ represent the center of the filter with $x, y$ being the coordinates over its domain. Let $L(x, y)$ be the input matrix representing nine elements and their surrounding space (a square space including a $3 \times 3$ matrix of elements); let Equations $\mathrm{A} 1$ and $\mathrm{A} 2$ be the Gabor operators. By performing a simple dot product, $G \cdot L$, we produce two filter response values:

$$
G L_{e v}\left(x_{c}, y_{c} \mid \lambda, \theta\right)=\sum_{x, y} G_{e v}\left(x, y \mid \lambda, \theta, x_{c}, y_{c}\right) \cdot L(x, y)
$$

and

$$
G L_{o d}\left(x_{c}, y_{c} \mid \lambda, \theta\right)=\sum_{x, y} G_{o d}\left(x, y \mid \lambda, \theta, x_{c}, y_{c}\right) \cdot L(x, y),
$$

where $x, y$ are indices over the basic matrix element $(x, y=0$ to three times the interelement distance, with 0 representing the top left corner of the input space). Finally, a simple squaring and summing of the convolutions of Equations $\mathrm{A} 3$ and $\mathrm{A} 4$ gives the combined filter energy level:

$$
E\left(x_{c}, y_{c} \mid \lambda, \theta\right)=G L_{e v}^{2}\left(x_{c}, y_{c} \mid \lambda, \theta\right)+G L_{o d}^{2}\left(x_{c}, y_{c} \mid \lambda, \theta\right) \text {. }
$$

Since Equation $A 5$ produces only a locally shift invariant response, we add a spatial integration stage (over $x_{c}, y_{c}$ ) to obtain a "texture energy" measure:

$$
T_{x, y}(\lambda, \theta)=\sum_{x_{c}, y_{c} \in S(x, y)} E\left(x_{c}, y_{c} \mid \lambda, \theta\right),
$$

where $x_{c}, y_{c}$ are indices indicating the center of the filter, and $S(x, y)$ is some excitatory neighborhood corresponding to a smoothing operation. This neighborhood is defined so as to ensure spatial invariance, and, hence, energy levels from 25 different filters are summed, with filter centers separated from each other equally $(5 \times 5$ matrix) within the space containing only the center element. This texture energy, $T$, represents the shift invariant response of the system. It should be noted that, although this sampling rate was arbitrarily chosen, using larger and smaller sampling rates produced similar results, and, thus, this choice has no bearing on the analysis. Each vertex in the curves of Figure 1 represents $T(\lambda, \theta)$ for that particular orientation and spatial frequency. (These three-dimensional curves actually use only a $1 \times 1$ input matrix; however, for our analysis, both inputs can at times produce similar qualitative results.) It should be noted that, as in the Rubenstein and Sagi (1990) model, Equations A2 and A2 assume equal sensitivity to all spatial frequencies. In the present analysis, we introduce a modulation transfer function (MTF) sensitivity coefficient (an inverted parabola, with a peak at $4 \mathrm{cy}-$ cles per degree; although this is approximate, since most frequencies used were smaller than the peak frequency, this curve is quite precise) for each energy $(E)$ value, dependent on the particular spatial frequency of the filter. This coefficient is not critical to the analysis, but was implemented nonetheless.

The next step is to average responses across orientation. This is desired because determining $\bar{\lambda}$ is independent of element orientation. However, because the $\Delta$ and $K$ produce textures with the same second-order statistics, performing this average will produce values that are the same for both, and, thus, discrimination will be impossible. To avoid this, and diverging from the Rubenstein and Sagi model, we introduce another nonlinearity (a squaring, see Equation A7), acting on the texture energy, $T$. Note that, at this point, energies have already been summed across space (neighborhood $S$ ). A nonlinearity at this stage represents a second-stage computa- 
tion, which according to Rubenstein and Sagi (1993) is feasible, considering their proposed second-stage filter that acts within orientation maps in a Gaussian excitatory way. Thus, for each spatial frequency, the response is:

$$
R(\lambda)=\sum_{\theta} T^{2}(\lambda, \theta)
$$

where increments of $45^{\circ}$ were used for orientation (using smaller increments also produced similar results). The final step is to obtain the response weighted average of the filter wavelength. This is simply a weighted average across the spatial-frequency spectrum as shown in Equation 1. Eight wavelengths were used, ranging from one quarter to two times the interelement distance (corresponding to a $1 \times 1$ input matrix, see details below). Like Equation A7, this represents a recombination stage. It should be noted that, in order to ensure that elements with iso-second-order statistics produce different responses, the nonlinearity in Equation A7 could have alternatively been acted on the first-stage energy response, $E$. However, we found that this design produced worse correlation to the psychophysics, compared with the analysis described here.

Using Pairs 1.1-1.8 from Experiment 1, each element was inputted and the results of filter analysis are depicted under each element in Figure 3. Notice that, for Pairs 1.1-1.4, the $K$ always has the larger $\bar{\lambda}$, in agreement with psychophysics. For Pairs 1.5 and 1.6, $\bar{\lambda}$ is approximately the same for both elements in their respective pair, and data show that these tasks are quite difficult. Pair 1.7 has texture elements with approximately the same texsize, but considering that these elements are no longer isoenergy, discrimination is still expected. Analysis of Pair 1.8 indicates that the $O$ has a larger $\bar{\lambda}$ than does the $C$, even though in Experiment 1 the elements were indiscriminable and in Experiment 2 the $C$ was the more visible of the two. Since these elements are not isoenergy, have different luminance values, have different orientation information (the $O$ has none, whereas the $C$ has a particular orientation represented), and Experiment 3 has shown that attention is involved with this task, texsize can no longer be used as the only indicator of discriminability.

Figure 17 summarizes these results by showing the correlation between performance thresholds and the ratio between $\bar{\lambda}$ s for each element of the pair. Notice that, when the smaller texsize texture was in the foreground (left half of graph), the threshold was high relative to when the larger of the pair was in the foreground (right half of graph; notice that all dotted lines have negative slopes). Note that, as the texsize difference increases, there is a reduction in performance asymmetry, indicating that texsize asymmetry is a threshold phenomenon.

One final note is that the actual input to the analysis for elements of Experiment 1 was a $1 \times 1$ matrix instead of the $3 \times 3$ neighborhood described above. Although both inputs produced similar qualitative results, we found that using a $1 \times 1$ matrix was sufficient for a few reasons. Comparing the ratio of element width to interelement distance with those in Experiment 7, we found that the ratio in Experiment 1 was similar to that of Pair 7.1. In the case of Pair 7.1, psychophysics showed that the neighboring elements had little influence on performance, and it was only when the ratio increased (i.e., Pairs 7.2-7.4) that performance levels changed. Also, taking into account all possible permutations for the $3 \times 3$ matrix, one must consider four different orientations and $13^{2}$ jittered positions for each element. This results in $\left(4 \times 13^{2}\right)^{9}=2.9 \times 10^{25}$ permutations. Even if each $\bar{\lambda}$ took $1 \mathrm{msec}$ to compute, years would be required to complete the simulation (the same demise would apply for only four orientations and four jittered positions, necessitating 2 years). Hence, each $\bar{\lambda}$ is a value averaged over 100 iterations (with each iteration randomly generated). After computing numerous values using these criteria, it was found that jittered inputs of the same element type produced results that varied quite considerably from one to the next, whereas those for nonjittered inputs had quite stable results. Consequently, analyses using the $3 \times 3$ input matrix were implemented with nonjittered elements. However, for elements of Experiment 1 using the 1 $X 1$ input matrix, a full simulation was performed.

All experiments studying the importance of texsize as a stimulus property (Experiment 1 and Experiments 4-10) are summarized in Figure 18. For each tested pair, the ratio of the thresholds is compared with the ratio between $\bar{\lambda}$ s for elements of that pair. Both ratios were computed by dividing data of the first element of the pair by the second element. Accordingly, if psychophysical data correlate with filter analysis, then all points should fall in either the top left or the bottom right quadrant. (Remember if the ratio of $\bar{\lambda} s$ is greater than one, then the ratio of the thresholds should be less than one, or vice versa.) For most pairs, this is the case. Notice that when $\bar{\lambda}$ is the same for both elements (ratio $=1$ ), then the ratio of thresholds should also be equal (see overlapping square symbols at cross hairs, representing Pairs 1.5 and 1.6 with randomly oriented elements). Also note that if performance asymmetry were predicted by element size (when defined as the minimal enclosing ellipse), then all points would be in the top left quadrant (since the size of element 1 in the figure was always the smaller of the pair). Those points not inside the top left or bottom right quadrants are represented by filled symbols. For Experiment 1, the filled squares represent data for Pairs 1.5 and 1.6 (see Figure 3), with elements of fixed orientation. As mentioned earlier, this stimulus gives rise to the square blobs arranging themselves in an orderly matrix. Upon inspection of these stimuli, this arrangement can be isolated as a transparent object, separate from the elements. Until now, no evidence has supported the extraction (and separation) of a transparent object at the preattentive level. If this is the case, then the task would become simply the original $\Delta / K$ task, as the data suggest.

For Experiments 5 and 6, the filled triangles represent data from Pairs 5.3 and 6.3 (see Figure 9). Upon inspection of the psychophysical data, it appears that performance asymmetry is not stable, with both asymmetries represented. Unlike other pairs, filter analysis of this pair using a slightly different range of wavelengths showed a flip in the $\bar{\lambda}$ ratio, indicating that perhaps different wavelength ranges (or criteria) were used by each of 
the observers during this task. This argument is relevant considering that, within each block, a stimulus type remained constant; also, it was observed that when an observer moved from a large-scale task to a small one, performance suffered for approximately 10 trials. This latter remark implies that observers may focus in on different parts of the frequency spectrum (i.e., different sets of wavelengths) when performing a particular task. Thus, using only one set of wavelengths for analysis of all experiments is not necessary. (For analysis using the $3 \times$ 3 input matrix, wavelengths used ranged from one quarter to 1.75 times the interelement distance, slightly different from the other $1 \times 1$ input matrix set.)

The remaining exceptions (filled circles) concern Pairs 10.2 and 10.3 of Experiment 10 (see Figure 16). In these cases, although filter analysis indicated the + as the larger $\bar{\lambda}$ element (and hence should be more visible), the energy variability across the orientation spectrum of the $L$ appears to be still quite dominant (see Rubenstein \& Sagi, 1990). This variability results in lower visibility of the + (higher thresholds) and, thus, a reversal of performance asymmetry would not be expected until the texsize of the + texture is substantially larger than that of the $L$ (Pair 10.4).

One final comment concerns the texsize measure in general. Upon inspection of the actual $\bar{\lambda}$ values in Figures 8,11 , and 15 , it appears that the magnitude of $\bar{\lambda}$ is more similar to the interelement distance than to the actual size of the individual element. Thus, it must be stressed that, although texsize represents in some way the size of an individual element, it is heavily influenced by the interelement distance and, hence, represents a more global measure of the stimulus.

(Manuscript received March 15, 1994;

revision accepted for publication August 28, 1995.) 